\title{
A note on D0-branes and instantons in $5 d$ supersymmetric gauge theories
}

\author{
Eran Avraham and Oren Bergman \\ Department of Physics, Technion, Israel Institute of Technology, \\ Haifa, 32000, Israel \\ E-mail: eranav@post.bgu.ac.il, bergman@physics.technion.ac.il
}

ABSTRACT: We refine a previous proposal for obtaining the multi-instanton partition function from the supersymmetric index of the $1 \mathrm{~d}$ supersymmetric gauge theory on the worldline of D0-branes. We provide examples where the refinements are crucial for obtaining the correct result.

Keywords: Brane Dynamics in Gauge Theories, Field Theories in Lower Dimensions, Supersymmetric Gauge Theory

ARXIV EPRINT: 1808.05648 


\section{Contents}

1 Introduction 1

2 General structure of $\mathcal{N}=(0,4)$ supersymmetric QM 4

3 Comparison with known results 5

3.1 The $\mathcal{N}=2$ theories

3.2 The $\operatorname{Sp}(N)+\boxminus$ theory 8

4 The $\mathrm{SU}(N)+2 \boxminus$ theory $\quad 9$

4.1 Adding flavors 11

$\begin{array}{ll}4.2 \text { A consistency check } & 12\end{array}$

$5 \quad$ The $\operatorname{Sp}\left(N_{1}\right) \times \operatorname{Sp}\left(N_{2}\right)+(\square, \square)$ theory 13

$\begin{array}{ll}5.1 \text { A consistency check } & 16\end{array}$

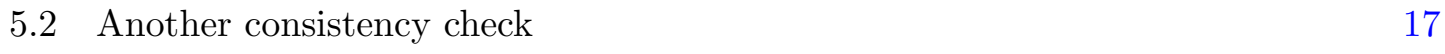

$\begin{array}{ll}5.3 \text { Adding flavors } & 17\end{array}$

$\begin{array}{llr}6 & \text { Conclusions } & 19\end{array}$

A The $\mathcal{N}=(0,4)$ index $\quad 19$

$\begin{array}{lll}\text { A.1 } & \operatorname{Sp}(N)+\boxminus & 21\end{array}$

A.2 $\mathrm{SU}(N)+2 \boxminus \quad 22$

A.3 $\operatorname{Sp}\left(N_{1}\right) \times \operatorname{Sp}\left(N_{2}\right)+(\square, \square) \quad 23$

B $\mathrm{SU}(2) \times \mathrm{Sp}(2)$ in the $\mathrm{SU}(N)$ formalism $\quad 24$

\section{Introduction}

The problem of counting instantons in supersymmetric gauge theories with eight supersymmetries has received some recent interest due to its appearance in the study of fivedimensional and six-dimensional superconformal field theories. In many cases these theories correspond to UV fixed points of $5 \mathrm{~d} \mathcal{N}=1$ supersymmetric gauge theories, in which the instantons, which are particles in five dimensions, provide a crucial ingredient in identifying the properties of the UV theory. To facilitate this we must be able to correctly compute the charges and degeneracies of the instanton states, namely to "count" instantons. In order to do this we must quantize the multi-instanton moduli space as provided by the ADHM construction [1]. In general this is a difficult problem since the moduli space contains singularities corresponding to instantons of vanishing size. Another way to say this 
is that the corresponding supersymmetric linear sigma model [2] is not well defined in the $\mathrm{UV}$, and requires a UV completion.

There have been a number of approaches for dealing with these singularities. Originally, Nekrasov used the so-called Omega-deformation to remove the singularities and compute the instanton partition function [3]. However this approach only works for $\mathrm{U}(N)$. Subsequently, Nekrasov and Shadchin were able to overcome the singularities for other gauge groups by lifting to five dimensions $[4,5]$.

An alternative approach is to find a UV completion of the ADHM sigma model, and attempt to extract from it the relevant data of the instanton moduli space. This approach is strongly motivated by string theory, in which five-dimensional supersymmetric gauge theories may be realized in terms of D4-branes in Type IIA superstring theory, and the instantons correspond to D0-branes inside the D4-branes [6]. The ADHM sigma model is naturally embedded in the $1 \mathrm{~d} \mathcal{N}=(0,4)$ supersymmetric gauge theory on the D0-brane worldline. It is the low energy effective theory on the Higgs branch of the gauge theory. On the other hand the $1 \mathrm{~d} \mathcal{N}=(0,4)$ gauge theory is a UV complete theory.

Recent advances in supersymmetric localization have made it possible to compute the exact partition function, or index, of such theories [7-10]. The multi-instanton partition function is then naively given by summing over instanton sectors:

$$
Z_{\mathrm{QM}}=\sum_{k=0}^{\infty} I_{k} q^{k},
$$

where $I_{k}$ is the index of the $1 \mathrm{~d} \mathcal{N}=(0,4)$ gauge theory of $k$ instantons, and $q$ is the instanton fugacity. The index $I_{k}$ is computed using supersymmetric localization, which reduces the path integral to ordinary contour integrals over the complexified holonomies of the gauge multiplet. The general form of the multi-particle index is given by the plethystic exponent of the single-particle index $f(q)$ (which will in general get contributions from multi-instanton bound states):

$$
Z_{\mathrm{QM}}=\mathrm{PE}[f(q)]=\exp \left[\sum_{n=1}^{\infty} \frac{f\left(q^{n}\right)}{n}\right] .
$$

By comparing with (1.1), one can extract information about the spectrum of one-particle states, up to a given instanton number $k$. Indeed one can often guess the complete $f(q)$ by computing $I_{k}$ for a small number of $k$ 's. This was done for the $\mathcal{N}=2$ supersymmetric $5 \mathrm{~d}$ $\mathrm{U}(N)$ gauge theory in [7], and used to show the existence of instanton bound states at all instanton numbers, as required by the conjecture that this theory flows to the $6 \mathrm{~d}$ A-type $(2,0)$ theory in the UV.

However, more generally one has to overcome the following difficulty. The moduli space of the 1d gauge theory generically also has additional branches that contribute to the index. For $\mathcal{N}=2$ theories the $1 \mathrm{~d}$ gauge theory has a Coulomb branch, and for $\mathcal{N}=1$ theories it can have both a Coulomb branch and a twisted Higgs branch parameterized by a twisted hypermultiplet. In the brane description these branches correspond to moving the D0-branes out of and away from the D4-branes. The contribution of the states on the 
extra branches must be removed in order to obtain the instanton partition function, which is just the contribution of the states on the Higgs branch. It seems natural to conjecture that the contribution of the extra states is accounted for by the reduced 1d gauge theory of the D0-branes in the absence of the D4-branes, namely

$$
Z_{\text {extra }}=Z_{\mathrm{QM}, \mathrm{red}}
$$

where $Z_{\mathrm{QM} \text {,red }}$ is computed for the $1 \mathrm{~d}$ gauge theory without the D4-branes, and

$$
Z_{\text {inst }}=\frac{Z_{\mathrm{QM}}}{Z_{\text {extra }}}
$$

This was originally conjectured in [8] for the class of $\mathcal{N}=1$ theories with gauge group $\operatorname{Sp}(N), N_{F} \leq 7$ flavor hypermultiplets, and an antisymmetric hypermultiplet, that are realized in terms of D4-branes in the background of an orientifold 8-plane and $N_{f}$ D8branes [11]. It was shown in [8], by computing the 1-instanton contribution, that this correctly reveals the enhanced exceptional global symmetries for $N_{F} \leq 5$. Subsequently $Z_{\text {extra }}$ was computed to higher orders in these theories and an all-orders expression was conjectured in [9]. In particular this completed the work of [8] and showed the enhanced $E_{7}$ and $E_{8}$ symmetries for $N_{F}=6$ and $N_{F}=7$, respectively.

The same issue was addressed in the $\mathcal{N}=2$ theories realized in terms of D4-branes in flat space or in the background of an orientifold 4-plane in [10]. For $\mathrm{U}(N)$ it was shown that $Z_{\text {extra }}=1$, in line with the result of [7], which did not include this correction. However for $\mathrm{O}(N)$ and $\operatorname{Sp}(N)$ the contribution of the extra branches is non-trivial. Furthermore, for $\operatorname{Sp}(N)$ it was found that an additional correction is required; in this case $Z_{\text {extra }}$ overcounts by a multiplicitive quantity, which corresponds to bound states of D0-branes that are confined to the orientifold 4-plane. This last observation can be understood from the fact that the reduced $1 \mathrm{~d}$ gauge theory in this case still has a remnant of the original Higgs branch, whose states we do not want to remove. The additional correction adds these contributions back in.

We would like to formalize this into the following modified proposal for the contribution of the extra states:

$$
Z_{\text {extra }}=\frac{Z_{\mathrm{QM}, \text { red }}}{\operatorname{PE}\left[f_{D 0_{c}}(q)\right]}
$$

where $f_{D 0_{c}}(q)$ is the single-particle index of D0-branes that are confined to any $5 \mathrm{~d}$ defect that remains after the 4-branes are removed. In addition, instead of simply removing the D4-branes by setting $N=0$ in the $1 \mathrm{~d}$ gauge theory, the reduced theory should be understood as the low-energy effective theory that results by turning on a mass for the fields charged under the 4-brane gauge symmetry and integrating them out. Geometrically this corresponds to moving the 4-branes away from the 0-branes. Depending on the theory, this can shift the values of Chern-Simons (CS) or discrete theta parameters. In all the examples studied in [7-10] the net shifts vanish, but, as we will see, in general they do not.

In this note we will present additional examples of $5 \mathrm{~d} \mathcal{N}=1$ gauge theories in support of our conjecture (1.5). We will consider the theories obtained by a $\mathbb{Z}_{2}$ orbifold of the 


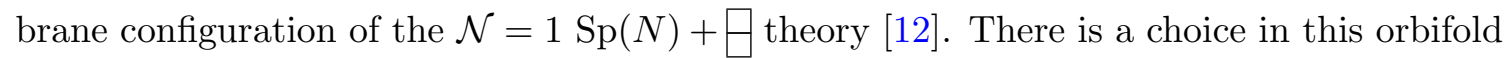
corresponding to the action of worldsheet parity in the twisted sector. The so-called orbifold with vector structure leads to the quiver theory with $\operatorname{Sp}\left(N_{1}\right) \times \operatorname{Sp}\left(N_{2}\right)$ and a bi-fundamental hypermultiplet, as well as flavors charged under either gauge group. The orbifold without vector structure gives an $\mathrm{SU}(N)$ gauge group with two antisymmetric hypermultiplets, plus the flavors. As we will see, these two examples exhibit the need both for the second correction factor in (1.5), as well as for the shift in the CS or discrete theta parameters in the reduced theory.

The outline for the rest of the paper is as follows. In section 2 we will review the general structure of $1 \mathrm{~d} \mathcal{N}=(0,4)$ gauge theories. In section 3 we will review the results for $Z_{\text {extra }}$ in the $5 \mathrm{~d} \mathcal{N}=2$ theories and $\mathcal{N}=1 \operatorname{Sp}(N)+\boxminus$ theory, and show that they are consistent with our proposal. In sections 4 and 5 we will apply our proposal to the $\mathbb{Z}_{2}$ orbifold theories, and perform some basic checks. Section 6 contains our conclusions.

\section{General structure of $\mathcal{N}=(0,4)$ supersymmetric QM}

A $1 \mathrm{~d}$ theory with $\mathcal{N}=(0,4)$ supersymmetry has four supercharges $Q^{\dot{\alpha} A}$ transforming in the $(\mathbf{2}, \mathbf{2})$ representation of $\mathrm{SO}(4)_{R}=\mathrm{SU}(2)_{r} \times \mathrm{SU}(2)_{r^{\prime}} .{ }^{1}$ This kind of supersymmetry admits four types of supermultiplets: a vector multiplet $\left(A_{0}, \varphi, \lambda^{\dot{\alpha} A}\right)$, a hypermultiplet $\left(\phi_{\dot{\alpha}}, \psi_{A}\right)$, a twisted hypermultiplet $\left(\phi_{A}, \psi_{\dot{\alpha}}\right)$, and a Fermi multiplet $\psi$. In terms of $\mathcal{N}=(0,2)$ multiplets, the $(0,4)$ vector multiplet decomposes into a vector multiplet and two Fermi multiplets, and the hypermultiplet and twisted hypermultiplet each decompose into a chiral and an anti-chiral multiplet.

The $\mathcal{N}=(0,4)$ theory for instantons in a $5 \mathrm{~d} \mathcal{N}=1$ gauge theory also has an $\mathrm{SU}(2)_{\ell}$ global symmetry, such that $\mathrm{SU}(2)_{\ell} \times \mathrm{SU}(2)_{r}$ is the rotation symmetry of the 5 d theory. Furthermore, for $5 \mathrm{~d}$ gauge theories that are realized on D4-branes, possibly in the presence of D8 "flavor branes", the 1d (and 5d) theory has an additional SU(2) $\ell^{\prime}$ global symmetry (denoted $\mathrm{SU}(2)_{L}$ in [9]). The symmetry $\mathrm{SU}(2)_{\ell^{\prime}} \times \mathrm{SU}(2)_{r^{\prime}}$ corresponds to rotations in the directions transverse to the D4-branes and along the D8-branes. Table 1 summarizes the generic content of the theory in these cases. The subscripts $R$ and $L$ on the fermions denote their chirality in the lift to two dimensions. In the one-dimensional theory this corresponds to the spin. The top three rows show the $1 \mathrm{~d}$ fields originating from the $5 \mathrm{~d}$ gauge multiplet. The rest are associated with the $5 \mathrm{~d}$ matter hypermultiplets. A $5 \mathrm{~d}$ matter field in the fundamental representation gives a 1d Fermi multiplet $\xi$. Matter fields in higher tensor representations lead to the $1 \mathrm{~d}$ fields shown in the bottom three rows. The four scalar fields in $X$ correspond to the positions of the D0-branes along the D4-branes, and the four scalar fields in $Y$ together with the vector multiplet scalar $\varphi$ correspond to the positions of the D0-branes transverse to the D4-branes.

The schematic form of the scalar potential is given by (see for example [13-15])

$$
V=\operatorname{Tr}\left[\left([X, X]+q^{2}\right)^{2}+[Y, Y]^{2}+[X, Y]^{2}+[X, \varphi]^{2}+[Y, \varphi]^{2}+q^{2}\left(Y^{2}+\varphi^{2}\right)\right] .
$$

\footnotetext{
${ }^{1}$ We follow the conventions of [9], and use a dotted greek index for $\mathrm{SU}(2)_{r}$ and an undotted capital latin index for $\mathrm{SU}(2)_{r^{\prime}}$. The latter is denoted $\mathrm{SU}(2)_{R}$ in [9]. We reserve subscripts $R$ and $L$ for fermion spin.
} 


\begin{tabular}{|l|l|c|c|c|c|c|}
\hline$(0,4)$ multiplet & Fields & $\mathrm{SU}(2)_{\ell} \times \mathrm{SU}(2)_{r}$ & $\mathrm{SU}(2)_{\ell^{\prime}} \times \mathrm{SU}(2)_{r^{\prime}}$ & $G_{D 0}$ & $G_{D 4}$ & $G_{D 8}$ \\
\hline Vector & $A_{0}, \varphi$ & $(\mathbf{1}, \mathbf{1})$ & $(\mathbf{1}, \mathbf{1})$ & adj & $\mathbf{1}$ & $\mathbf{1}$ \\
& $\lambda_{R}$ & $(\mathbf{1}, \mathbf{2})$ & $(\mathbf{1}, \mathbf{2})$ & & & \\
\hline Hyper (real) & $X$ & $(\mathbf{2}, \mathbf{2})$ & $(\mathbf{1}, \mathbf{1})$ & $R_{X}$ & $\mathbf{1}$ & $\mathbf{1}$ \\
& $\chi_{L}$ & $(\mathbf{2}, \mathbf{1})$ & $(\mathbf{1}, \mathbf{2})$ & & & \\
\hline Hyper (complex) & $q$ & $(\mathbf{1}, \mathbf{2})$ & $(\mathbf{1}, \mathbf{1})$ & $\square$ & $\square$ & $\mathbf{1}$ \\
& $\psi_{L}$ & $(\mathbf{1}, \mathbf{1})$ & $(\mathbf{1}, \mathbf{2})$ & & & \\
\hline \hline Fermi & $\xi_{R}$ & $(\mathbf{1}, \mathbf{1})$ & $(\mathbf{1}, \mathbf{1})$ & $\square$ & $\mathbf{1}$ & $\square$ \\
\hline \hline Twisted Hyper & $Y$ & $(\mathbf{1}, \mathbf{1})$ & $(\mathbf{2}, \mathbf{2})$ & $R_{Y}$ & $\mathbf{1}$ & $\mathbf{1}$ \\
& $\lambda_{L}$ & $(\mathbf{1}, \mathbf{2})$ & $(\mathbf{2}, \mathbf{1})$ & & & \\
\hline Fermi & $\chi_{R}$ & $(\mathbf{2}, \mathbf{1})$ & $(\mathbf{2}, \mathbf{1})$ & $R_{\chi}$ & $\mathbf{1}$ & $\mathbf{1}$ \\
\hline Fermi & $\psi_{R}$ & $(\mathbf{1}, \mathbf{1})$ & $(\mathbf{2}, \mathbf{1})$ & $\square$ & $R_{\psi}$ & $\mathbf{1}$ \\
\hline
\end{tabular}

Table 1. Spectrum of $1 \mathrm{~d} \mathcal{N}=(0,4)$ gauge theory in a generic D0-D4-D8 system. The unspecified representations depend on the specific model being considered. The subscript $L, R$ refers to the spin of the corresponding fermion.

The theory generically has three distinct branches of vacua:

- A Higgs branch given by $\varphi=Y=0$ and $[X, X]+q^{2}=0$

- A Coulomb branch given by $q=0$ and $[X, X]=[Y, Y]=[X, Y]=[X, \varphi]=[Y, \varphi]=0$

- A twisted Higgs branch given by $\varphi=q=0$ and $[X, X]=[Y, Y]=[X, Y]=0$

There are also mixed branches, where some components of $\varphi$ vanish and some components of $q$ vanish. The reduced theory obtained by removing the fields $q, \psi_{L}$ and $\psi_{R}$ will clearly have the same Coulomb and twisted Higgs branches as the original theory. The main issue will be whether it also possesses a remnant of the original Higgs branch, namely whether the space defined by $\varphi=Y=[X, X]=0$ has components not contained in the Coulomb or twisted Higgs branches.

\section{Comparison with known results}

\subsection{The $\mathcal{N}=2$ theories}

The $5 \mathrm{~d} \mathcal{N}=2$ theories are completely characterized by the gauge group $G_{D 4}=\mathrm{U}(N)$, $\mathrm{O}(N)$, or $\operatorname{Sp}(N)$. In the $\mathcal{N}=1$ language these theories have a single matter hypermultiplet in the adjoint representation. The $\mathrm{U}(N)$ theory is realized on $N$ parallel D4-branes in Type IIA string theory. Adding an $\mathrm{O}^{-}$or $\widetilde{\mathrm{O}}^{-}$plane gives the $\mathrm{O}(N)$ theory with $N$ even or odd, respectively. Adding an $\mathrm{O}_{4}^{+}$or $\widetilde{\mathrm{O}}^{+}$plane gives the $\operatorname{Sp}(N)$ theory with the discrete theta parameter $\theta=0$ or $\pi$, respectively [16]. These theories have six-dimensional UV fixed points corresponding to 6d A-type or D-type $(2,0)$ theories described by M5-branes in $M$ theory. 


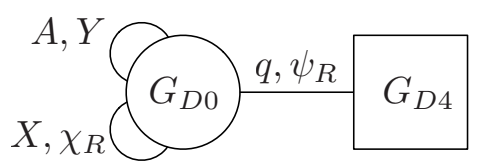

Figure 1. $1 \mathrm{~d} \mathcal{N}=(4,4)$ theories for the $5 \mathrm{~d} \mathcal{N}=2$ theories.

The D0-brane gauge theory in these cases is shown in figure 1, with $G_{D 0}=\mathrm{U}(k), \operatorname{Sp}(k)$, and $\mathrm{O}(k)$ when $G_{D 4}=\mathrm{U}(N), \mathrm{O}(N)$, and $\operatorname{Sp}(N)$, respectively. ${ }^{2}$ The twisted hypermultiplet $Y$ transforms in the adjoint representation of $G_{D 0}$, the multiplets $q$ and $\psi_{R}$ transform in the bifundamental of $G_{D 0} \times G_{D 4}$, and $X$ and $\chi_{R}$ transform in the adjoint, antisymmetric, and symmetric representation of $G_{D 0}$ in the three cases, respectively. These theories really have $\mathcal{N}=(4,4)$ supersymmetry: the vector multiplet and twisted hypermultiplet combine into a $(4,4)$ vector multiplet, and each hypermultiplet combines with a Fermi multiplet into a $(4,4)$ hypermultiplet. There is a Coulomb branch that combines the $\mathcal{N}=(0,4)$ Coulomb and twisted Higgs branches parameterized by $(\varphi, Y)$, and a Higgs branch parameterized by $(X, q)$.

The reduced $1 \mathrm{~d}$ gauge theories are given by removing the fields $\left\{q, \psi_{L}\right\}$ and $\psi_{R}$. The multi-particle indices corresponding to the reduced theories in the different cases were given in [10] as follows (the notation is reviewed in the appendix):

$$
Z_{\mathrm{QM}, \text { red }}= \begin{cases}1 & \mathrm{U}(N) \\ \operatorname{PE}\left[\frac{t^{2}\left(t+\frac{1}{t}\right)\left(v+\frac{1}{v}-u-\frac{1}{u}\right)}{2(1-t u)\left(1-\frac{t}{u}\right)(1+t v)\left(1+\frac{t}{v}\right)} \frac{q}{1-q}\right] & \mathrm{O}(N), N \text { even } \\ \operatorname{PE}\left[\frac{t^{2}\left(t+\frac{1}{t}\right)\left(v+\frac{1}{v}-u-\frac{1}{u}\right)}{2(1-t u)\left(1-\frac{t}{u}\right)(1+t v)\left(1+\frac{t}{v}\right)} \frac{-q}{1+q}\right] & \mathrm{O}(N), N \text { odd } \\ \operatorname{PE}\left[\frac{t^{2}\left(t+\frac{1}{t}\right)\left(v+\frac{1}{v}-u-\frac{1}{u}\right)}{2(1-t u)\left(1-\frac{t}{u}\right)(1+t v)\left(1+\frac{t}{v}\right)} \frac{q^{2}}{1-q^{2}}+\frac{t\left(v+\frac{1}{v}-u-\frac{1}{u}\right)}{(1-t u)\left(1-\frac{t}{u}\right)} \frac{q}{1-q^{2}}\right] & \mathrm{Sp}(N)_{0} \\ \operatorname{PE}\left[\frac{t^{2}\left(t+\frac{1}{t}\right)\left(v+\frac{1}{v}-u-\frac{1}{u}\right)}{2(1-t u)\left(1-\frac{t}{u}\right)(1+t v)\left(1+\frac{t}{v}\right)} \frac{-q^{2}}{1+q^{2}}+\frac{t\left(v+\frac{1}{v}-u-\frac{1}{u}\right)}{(1-t u)\left(1-\frac{t}{u}\right)} \frac{q^{2}}{1-q^{4}}\right] & \operatorname{Sp}(N)_{\pi}\end{cases}
$$

However it was argued in [10] that the second term in the one-particle index in the plethystic exponent for the $\operatorname{Sp}(N)$ theories should be discarded to agree with the M theory viewpoint. The contribution of the extra states was therefore claimed to be given by

$$
Z_{\text {extra }}= \begin{cases}Z_{\mathrm{QM}, \text { red }} & \mathrm{U}(N), \mathrm{O}(N) \\ Z_{\mathrm{QM}, \text { red }} \cdot\left(\mathrm{PE}\left[\frac{t\left(v+\frac{1}{v}-u-\frac{1}{u}\right)}{(1-t u)\left(1-\frac{t}{u}\right)} \frac{q}{1-q^{2}}\right]\right)^{-1} \mathrm{Sp}(N)_{0} \\ Z_{\mathrm{QM}, \text { red }} \cdot\left(\mathrm{PE}\left[\frac{t\left(v+\frac{1}{v}-u-\frac{1}{u}\right)}{(1-t u)\left(1-\frac{t}{u}\right)} \frac{q^{2}}{1-q^{4}}\right]\right)^{-1} \mathrm{Sp}(N)_{\pi}\end{cases}
$$

As we will now demonstrate these identifications are consistent with our proposal in (1.5).

\footnotetext{
${ }^{2}$ For the case of $G_{D 0}=\mathrm{U}(k)$ and $G_{D 4}=\mathrm{U}(N)$, the global symmetry of the $1 \mathrm{~d} \mathrm{U}(k)$ gauge theory is actually $\mathrm{SU}(N)$, since the $\mathrm{U}(1) \in \mathrm{U}(N)$ is gauged.
} 
Our proposal has two new ingredients. First, one must take into account the possible shifts in the values of the gauge theory parameters as a result of integrating out rather than just removing the massive fermions $\psi_{L}$ and $\psi_{R}$. This is relevant for the $1 \mathrm{~d} \mathrm{U}(k)$ and $\mathrm{O}(k)$ theories.

The $1 \mathrm{~d} \mathrm{U}(k)$ theory admits a $\mathrm{CS}$ term $\kappa \int \operatorname{Tr} A$, with $\kappa \in \mathbb{Z}$, that is inhereted from the CS term of the $5 \mathrm{~d} \mathrm{U}(N)$ theory (see for example [17]). This can be thought of as a background U(1) gauge charge of size $\kappa$. The effective CS parameter of the reduced theory will get a contribution from the massive fermions that are integrated out. Each fermion in the fundamental representation of $\mathrm{U}(k)$ contributes $\frac{1}{2} \operatorname{sign}(m)$, where sign of the mass is given by the product of the spin and the diagonal $\mathrm{U}(1)$ charge of the fermion. The net shift vanishes since the hypermultiplet fermion $\psi_{L}$ and the Fermi multiplet fermion $\psi_{R}$ have the same charge but opposite spin.

The $1 \mathrm{~d} \mathrm{O}(k)$ theory admits a discrete theta parameter $\theta$ taking values in $\{0, \pi\}$, that is inhereted from the discrete theta parameter of the $5 \mathrm{~d} \operatorname{Sp}(N)$ theory [18]. This can be regarded as a background $\mathrm{O}(1)=\mathbb{Z}_{2}$ gauge charge. The effective theta parameter of the reduced theory will get a contribution depending on the sign of the determinant of the fermion mass matrix [22]. The mass-deformation moving the 4-branes breaks $\operatorname{Sp}(N)$ to $\mathrm{U}(N)$. The fermions $\psi_{R}$ and $\psi_{L}$ each decompose into $N$ states carrying positive charge under the diagonal $\mathrm{U}(1)$, and $N$ carrying negative charge. Therefore there are $2 N$ fermions of negative mass and $2 N$ of positive mass, and therefore no shift in $\theta$.

The second ingredient of our proposal has to do with the overcounting of extra states when the moduli space of the reduced theory contains a remnant of the Higgs branch of the original theory. Equivalently, the question is whether the reduced theory has a Higgs branch that is separate from its Coulomb branch. The would-be Higgs branch of the reduced theory is given by $\varphi_{H}=Y_{H}=0$ and

$$
X_{H}= \begin{cases}\operatorname{diag}\left[x_{1}, \ldots, x_{k}\right] & \mathrm{U}(k) \\ \operatorname{diag}\left[x_{1} \sigma_{2}, \ldots, x_{k} \sigma_{2}\right] & \mathrm{Sp}(k) \\ \operatorname{diag}\left[x_{1}, \ldots, x_{2 n}\right] & \mathrm{O}(k=2 n) \\ \operatorname{diag}\left[x_{1}, \ldots, x_{2 n+1}\right] & \mathrm{O}(k=2 n+1) .\end{cases}
$$

On the other hand on the Coulomb branch

$$
\varphi_{C}= \begin{cases}\operatorname{diag}\left[a_{1}, \ldots, a_{k}\right] & \mathrm{U}(k) \\ \operatorname{diag}\left[a_{1} \mathbb{I}, \ldots, a_{k} \mathbb{I}\right] & \mathrm{Sp}(k) \\ \operatorname{diag}\left[a_{1} \sigma_{2}, \ldots, a_{n} \sigma_{2}\right] & \mathrm{O}(2 n) \\ \operatorname{diag}\left[a_{1} \sigma_{2}, \ldots, a_{n} \sigma_{2}, 0\right] & \mathrm{O}(2 n+1)\end{cases}
$$

with $Y_{C}$ having the same form, and

$$
X_{C}= \begin{cases}\operatorname{diag}\left[x_{1}, \ldots, x_{k}\right] & \mathrm{U}(k) \\ \operatorname{diag}\left[x_{1} \sigma_{2}, \ldots, x_{k} \sigma_{2}\right] & \mathrm{Sp}(k) \\ \operatorname{diag}\left[x_{1} \mathbb{I}, \ldots, x_{n} \mathbb{I}\right] & \mathrm{O}(2 n) \\ \operatorname{diag}\left[x_{1} \mathbb{I}, \ldots, x_{n} \mathbb{I}, x\right] & \mathrm{O}(2 n+1)\end{cases}
$$




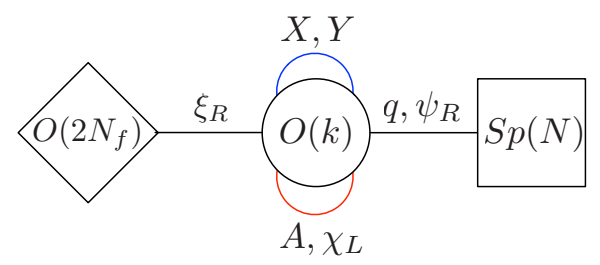

Figure 2. The $1 \mathrm{~d} \mathcal{N}=(0,4)$ theory for the $5 \mathrm{~d} \operatorname{Sp}(N)$ with an antisymmetric hypermultiplet and $N_{f}$ fundamental hypermultiplets.

We see that only the orthogonal theories have a separate Higgs branch. This can be understood as the possibility for a bulk D0-brane to split into a pair of fractional D0branes confined to an $\mathrm{O}^{+}$-plane. The split phase, in which the two fractional D0-branes are restricted to moving on the orientifold plane, is a remnant of the original Higgs branch, in which the D0-branes were inside the D4-branes. This splitting is not possible on an $\mathrm{O} 4^{-}$-plane, which is why there is no Higgs branch in the reduced $\operatorname{Sp}(k)$ theory.

Our proposal is therefore consistent with the identification of $Z_{\text {extra }}$ for the $5 \mathrm{~d} \mathrm{U}(N)$ and $\mathrm{O}(N)$ theories in (3.2). For the $\operatorname{Sp}(N)$ theory we need to divide by the correction factor corresponding to confined D0-branes, $\mathrm{PE}\left[f_{D 0_{c}}(q)\right]$. The quantity $f_{D 0_{c}}(q)$ is the one-particle index for D0-branes confined to the $\mathrm{O}^{+}{ }^{+}$-plane. This quantity was originally studied in [19]. The $\mathcal{O}(q)$ term is just the index of the reduced $1 \mathrm{~d}$ theory with $k=1$, so

$$
f_{D 0_{c}}(q)=\frac{1}{2}\left(1+e^{i \theta}\right) I_{1}^{X} I_{1}^{\chi_{R}} q+\mathcal{O}\left(q^{2}\right)=\frac{1}{2}\left(1+e^{i \theta}\right) \frac{t\left(u+\frac{1}{u}-v-\frac{1}{v}\right)}{(1-t u)\left(1-\frac{t}{u}\right)} q+\mathcal{O}\left(q^{2}\right)
$$

This reproduces $\mathcal{O}(q)$ term in the correction factor for $\theta=0$ in (3.2). The higher order terms are more difficult to obtain. For $\theta=\pi$ the single D0-brane state is projected out due to the background $\mathbb{Z}_{2}$ charge, and the lowest order contribution is at $\mathcal{O}\left(q^{2}\right)$, in agreement with (3.2). This corresponds to a state with two confined D0-branes connected by a string which cancels the background charge.

\subsection{The $\operatorname{Sp}(N)+\square$ theory}

This is the theory on $N$ D4-branes in an $\mathrm{O}^{-}$-plane with $N_{f}$ D8-branes. For $N_{f} \leq 7$ this theory has a $5 \mathrm{~d}$ UV fixed point with an enhanced $E_{N_{f}+1}$ global symmetry [11]. For $N_{f}=8$ it corresponds to the $6 \mathrm{~d}(1,0)$ E-string theory. For $N_{f}=0$ there is another possibility associated to the choice of discrete theta parameter, the so-called $\tilde{E}_{1}$ theory, in which the global U(1) symmetry is not enhanced [20-22]. For $N_{f}>0$ the theta parameter is not physical, since it can be removed by a transformation in the parity-reversing component of the global $\mathrm{O}\left(2 N_{f}\right)$ symmetry.

The corresponding $1 \mathrm{~d} \mathcal{N}=(0,4)$ gauge theory on the D0-branes is shown in figure 2. The hypermultiplet $X$ and twisted hypermutiplet $Y$ both transform in the symmetric representation of $\mathrm{O}(k)$. The reduced 1d gauge theory is again given by removing the fields $\left\{q, \psi_{L}\right\}$ and $\psi_{R}$. The multi-particle indices corresponding to the reduced theory with 
$0 \leq N_{f} \leq 8$ were given in [9] as follows:

$$
Z_{\mathrm{QM}, \mathrm{red}}= \begin{cases}\mathrm{PE}\left[\frac{-t^{2}}{(1-t u)\left(1-\frac{t}{u}\right)(1-t v)\left(1-\frac{t}{v}\right)} q\right] & N_{f}=0 \\ \mathrm{PE}\left[\frac{-t^{2}}{(1-t u)\left(1-\frac{t}{u}\right)(1-t v)\left(1-\frac{t}{v}\right)} q \chi[f]_{\mathbf{2}^{N_{f}-1}}^{\mathrm{O}\left(2 N_{f}\right)}\right] & N_{f}=1, \ldots, 5 \\ \mathrm{PE}\left[\frac{-t^{2}}{(1-t u)\left(1-\frac{t}{u}\right)(1-t v)\left(1-\frac{t}{v}\right)}\left(q \chi[f]_{\mathbf{3 2}}^{\mathrm{O}(12)}+q^{2}\right)\right] & N_{f}=6 \\ \mathrm{PE}\left[\frac{-t^{2}}{(1-t u)\left(1-\frac{t}{u}\right)(1-t v)\left(1-\frac{t}{v}\right)}\left(q \chi[f]_{\mathbf{6 4}}^{\mathrm{O}(14)}+q^{2} \chi[f]_{\mathbf{1 4}}^{\mathrm{O}(14)}\right)\right] & N_{f}=7 \\ \mathrm{PE}\left[\frac{-\left(t+t^{3}\right)\left(u+\frac{1}{u}+v+\frac{1}{v}\right)}{2(1-t u)\left(1-\frac{t}{u}\right)(1-t v)\left(1-\frac{t}{v}\right)} \frac{q^{2}}{1-q^{2}}\right. & \\ \left.-\frac{t^{2}}{(1-t u)\left(1-\frac{t}{u}\right)(1-t v)\left(1-\frac{t}{v}\right)}\left(\chi[f]_{\mathbf{1 2 0}}^{\mathrm{O}(16)} \frac{q^{2}}{1-q^{2}}+\chi[f]_{\mathbf{1 2 8}}^{\mathrm{O}(16)} \frac{q}{1-q^{2}}\right)\right] & N_{f}=8\end{cases}
$$

where $f$ denotes collectively the flavor fugacities. In all cases these were claimed to give precisely the extra factors, namely

$$
Z_{\text {extra }}=Z_{\mathrm{QM}, \text { red }} \cdot
$$

As a slight generalization we also give the result for $N_{f}=0$ and $\theta=\pi$ (which we checked to $\left.\mathcal{O}\left(q^{4}\right)\right)$ :

$$
Z_{\text {extra }}\left[N_{f}=0, \theta=\pi\right]=Z_{\mathrm{QM}, \mathrm{red}}\left[N_{f}=0, \theta=\pi\right]=1 .
$$

These results are also consistent with our proposal. First we observe that the net shift in the discrete theta parameter in the $N_{f}=0$ theory vanishes since there are an even number $2 N$ of negative mass fermions. As to the moduli space of the reduced theory, on the Coulomb branch $\varphi_{C}$ has the same form as in (3.4) for the orthogonal theory, and $X_{C}$ and $Y_{C}$ take the same form as in (3.5) for the orthogonal theory. There is also a twisted Higgs branch, on which $\varphi_{T H}=0$ and

$$
\begin{aligned}
Y_{T H} & =\operatorname{diag}\left[y_{1}, \ldots, y_{k}\right] \\
X_{T H} & =\operatorname{diag}\left[x_{1}, \ldots, x_{k}\right] .
\end{aligned}
$$

The would-be Higgs branch of the reduced theory is given by $\varphi_{H}=Y_{H}=0$ and $X_{H}=$ $\operatorname{diag}\left[x_{1}, \ldots, x_{k}\right]$, and is therefore completely contained in the twisted Higgs branch. There is no separate Higgs branch in this case, and therefore no correction factor. The reduced theory precisely accounts for the extra states.

\section{$4 \quad$ The $\mathrm{SU}(N)+2 \boxminus$ theory}

This theory is obtained from the $\mathbb{Z}_{2}$ orbifold without vector structure of the $\operatorname{Sp}(N)+\boxminus$ theory. The corresponding $1 \mathrm{~d} \mathcal{N}=(0,4)$ gauge theory for $k$ D0-branes in this case is shown in figure 3 . There is an additional $\mathrm{U}(1)_{\ell^{\prime}}$ global symmetry in this case, since the symmetry rotating the two antisymmetrics is $\mathrm{U}(2)_{\ell^{\prime}}=\mathrm{SU}(2)_{\ell^{\prime}} \times \mathrm{U}(1)_{\ell^{\prime}}$. The $\mathrm{SU}(2)_{\ell^{\prime}}$ doublets, $Y, \chi_{R}$, 


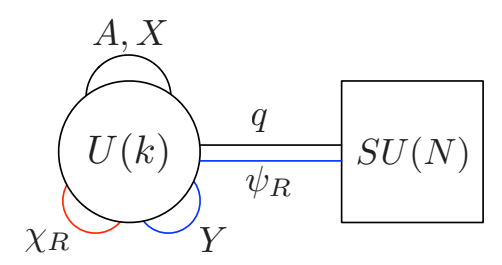

Figure 3. (a) The $1 \mathrm{~d} \mathcal{N}=(0,4)$ theory for the $5 \mathrm{~d} \mathrm{SU}(N)$ with two anisymmetrics.

and $\psi_{R}$, all carry one unit of charge under $\mathrm{U}(1)_{\ell^{\prime}}$. All other mutiplets are neutral under this symmetry. The twisted hypermultiplet $Y$ transforms in the symmetric of $\mathrm{U}(k)$, the Fermi multiplet $\chi_{R}$ transforms in the antisymmetric, the hypermultiplet $q$ transforms in $(\mathbf{k}, \overline{\mathbf{N}})$, and the Fermi multiplet $\psi_{R}$ in $(\mathbf{k}, \mathbf{N})$. Note in particular the different representations of $q$ and $\psi_{R}$. The $1 \mathrm{~d} \mathrm{U}(k)$ theory admits a $\mathrm{CS}$ term inhereted from the $5 \mathrm{~d} \mathrm{SU}(N)$ theory. As before, this corresponds to a background $\mathrm{U}(1)$ gauge charge $\kappa$. In addition there can be a background $\mathrm{U}(1)_{\ell^{\prime}}$ global charge $\zeta$, corresponding to a CS term for a background $\mathrm{U}(1)_{\ell^{\prime}}$ gauge field $\zeta \int A_{\ell^{\prime}}$. $^{3}$

Let us now consider the reduced theory obtained by removing $q$ and $\psi_{R}$. As in the $\mathcal{N}=2$ case in the previous section, the would-be Higgs branch of the reduced theory given by $\varphi_{H}=Y_{H}=0$ and $X_{H}=\operatorname{diag}\left[x_{1}, \ldots x_{k}\right]$ is fully contained in the Coulomb branch, and therefore does not include a remnant of the original Higgs branch. ${ }^{4}$ Therefore the reduced theory should correctly account for the extra states without the additional correction factor. Indeed we do not expect such a correction since the orbifold without vector structure does not support fractional D0-branes.

However the CS parameter $\kappa$ and the background $\mathrm{U}(1)_{\ell^{\prime}}$ charge $\zeta$ of the reduced theory are shifted from their values in the original theory:

$$
\Delta \kappa=2 \Delta \zeta=\left\{\begin{array}{rl} 
\pm 2 & N=2 n+1 \\
0 & N=2 n
\end{array}\right.
$$

We can see this as follows. For $N=2 n$, the mass deformation that moves the D4-branes away from the D0-branes, and specifically away from the O8-plane, breaks the 5 d symmetry $\mathrm{SU}(2 n)$ to $\mathrm{SU}(n) \times \mathrm{SU}(n) \times \mathrm{U}(1)$. The fermions $\psi_{R}$ and $\psi_{L}$ decompose as $(\mathbf{n}, \mathbf{1})_{+}+(\mathbf{1}, \mathbf{n})_{-}$ and $(\overline{\mathbf{n}}, \mathbf{1})_{-}+(\mathbf{1}, \overline{\mathbf{n}})_{+}$, respectively. Since the fermion mass is proportional to the $\mathrm{U}(1)$ charge and the spin, each one gives $N$ positive mass fermions and $N$ negative mass fermions. The CS parameter gets contributions from both $\psi_{R}$ and $\psi_{L}$, and the background global charge $\zeta$ gets contributions only from $\psi_{R}$. In either case the net shift vanishes since there are an equal number of negative and positive contributions. On the other hand for $N=2 n+1$ the unbroken 5 d symmetry is $\mathrm{SU}(n) \times \mathrm{SU}(n+1) \times \mathrm{U}(1)$, and the two fermions decompose as $(\mathbf{n}, \mathbf{1})_{+}+(\mathbf{1}, \mathbf{n}+\mathbf{1})_{-}$and $(\overline{\mathbf{n}}, \mathbf{1})_{-}+(\mathbf{1}, \overline{\mathbf{n}+\mathbf{1}})_{+}$. In this case there is a mismatch of positive and negative mass fermions which shifts $\kappa$ by $\Delta \kappa= \pm 2 \times 2 \times \frac{1}{2}= \pm 2$, and shifts $\zeta$

\footnotetext{
${ }^{3}$ This comes from a mixed CS term in $5 \mathrm{~d}$ coupling the $\mathrm{SU}(N)$ gauge field to a background $\mathrm{U}(1)_{\ell^{\prime}}$ gauge field, $\zeta \int A_{\ell^{\prime}} \wedge \operatorname{Tr}(F \wedge F)$. In the background of an instanton particle this reduces to $\zeta \int A_{\ell^{\prime}}$.

${ }^{4}$ In this case the would-be twisted Higgs branch is also contained in the Coulomb branch.
} 
by $\Delta \zeta= \pm 2 \times \frac{1}{2}= \pm 1$. The common factor of 2 is due to the fact that $\psi_{R}$ and $\psi_{L}$ are both doublets (of SU $(2)_{\ell^{\prime}}$ and $\mathrm{SU}(2)_{r^{\prime}}$, respectively). At this point it is difficult to fix the sign of the shift. However we will see below that the minus sign appears to be the correct one.

Our proposal for $Z_{\text {extra }}$ in this case is then given by

$$
Z_{\text {extra }}[\kappa, \zeta]=Z_{\mathrm{QM}, \operatorname{red}}\left[\kappa^{\prime}, \zeta^{\prime}\right]
$$

where $\kappa^{\prime}=\kappa+\Delta \kappa=\kappa \pm 2$ and $\zeta^{\prime}=\zeta+\Delta \zeta=\zeta \pm 1$. The one-instanton contribution to $Z_{\mathrm{QM}, \text { red }}$ is given by

$$
\begin{aligned}
& I_{1, \mathrm{red}}\left[\kappa^{\prime}, \zeta^{\prime}\right]=\frac{a^{\zeta^{\prime}}}{2 \pi i} \oint z^{\kappa^{\prime}} I_{1}^{A} I_{1}^{X} I_{1}^{Y}
\end{aligned}
$$

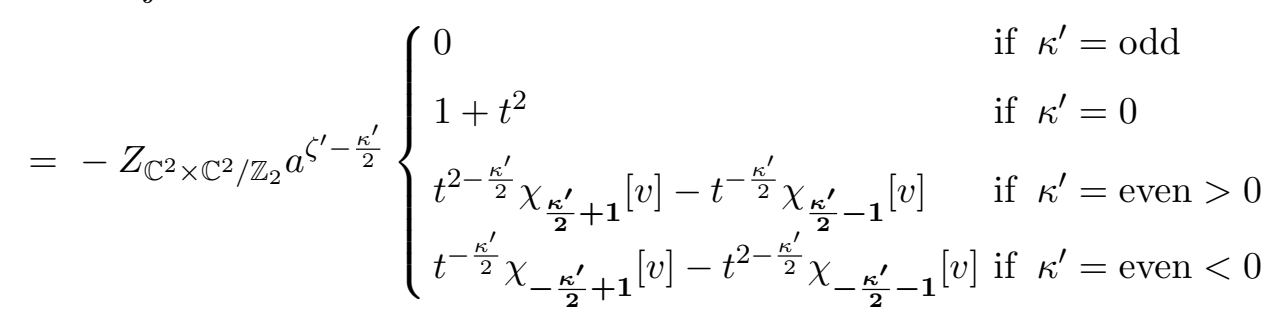

where $\chi_{\mathbf{d}}[v]$ is the character of the $d$-dimensional representation of $\mathrm{SU}(2)_{\ell^{\prime}}, a$ denotes the $\mathrm{U}(1)_{\ell^{\prime}}$ fugacity, and we have defined

$$
Z_{\mathbb{C}^{2} \times \mathbb{C}^{2} / \mathbb{Z}_{2}} \equiv \frac{t^{2}}{(1-t u)\left(1-\frac{t}{u}\right)\left(1-t^{2} v^{2}\right)\left(1-\frac{t^{2}}{v^{2}}\right)} .
$$

This factor is associated to the translational zero modes of a D0-brane moving in $\mathbb{C}^{2} \times$ $\mathbb{C}^{2} / \mathbb{Z}_{2}$. The one-instanton contribution vanishes for any odd value of $\kappa^{\prime}$ due to the nonvanishing background gauge charge. For even values of $\kappa^{\prime}$ the background charge can be cancelled by the $Y$ multiplet, resulting in a nonzero contribution.

We claim that the full multi-particle index of the reduced theory is given by

$$
Z_{\mathrm{QM}, \mathrm{red}}=\mathrm{PE}\left[q I_{1, \mathrm{red}}\right]
$$

namely that there are no D0-brane bound states. As a test of this claim we have computed the two-instanton contribution for $\kappa^{\prime}=0, \pm 2$ :

$$
I_{2, \text { red }}= \begin{cases}a^{2 \zeta^{\prime}}\left(\chi_{\mathbf{2}}[u] t^{5}+\left(1+\chi_{\mathbf{3}}[u]+\chi_{\mathbf{3}}[v]\right) t^{6}+\mathcal{O}\left(t^{7}\right)\right) & \text { for } \kappa^{\prime}=0 \\ a^{2 \zeta^{\prime}-2}\left(t^{6}+\chi_{\mathbf{2}}[u] \chi_{\mathbf{2}}[v]^{2} t^{7}+\mathcal{O}\left(t^{8}\right)\right) & \text { for } \kappa^{\prime}= \pm 2 .\end{cases}
$$

This agrees with the $q^{2}$ term in the expansion of (4.5) for $\kappa^{\prime}=0, \pm 2$.

\subsection{Adding flavors}

Adding $N_{f}$ fundamentals (flavors) to the 5 d theory adds $N_{f}$ Fermi multiplets $\xi_{R}^{i}$ to the $1 \mathrm{~d}$ $\mathcal{N}=(0,4)$ theory, figure 4 . These contribute an additional factor of the form

$$
I_{k}^{\xi_{R}}=\prod_{I=1}^{k}\left[z_{I}^{N_{f} / 2} \sum_{m=0}^{N_{f}}(-1)^{m} z_{I}^{-m} \chi_{[m]}[f]\right]
$$




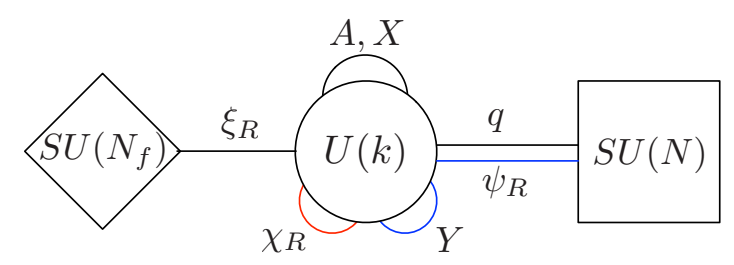

Figure 4. The $1 \mathrm{~d} \mathcal{N}=(0,4)$ theory for the $5 \mathrm{~d} \mathrm{SU}(N)$ with two anisymmetrics and $N_{f}$ fundamentals.

where $\chi_{[m]}$ denotes the character of the antisymmetric $m$-tensor representation of $\mathrm{SU}\left(N_{f}\right)$. Our proposal for $Z_{\text {extra }}$ is the same as in (4.2), namely $Z_{\text {extra }}[\kappa, \zeta]=Z_{\mathrm{QM}, \text { red }}\left[\kappa^{\prime}, \zeta^{\prime}\right]$. Here $\kappa$ (and therefore also $\kappa^{\prime}$ ) takes values in $\mathbb{Z}+N_{f} / 2$ due to the parity anomaly. We expect that for a small number of flavors (4.5) will continue to hold, namely that $Z_{\mathrm{QM} \text {,red }}=\operatorname{PE}\left[q I_{1, \text { red }}\right]$, where $I_{1 \text {,red }}$ is the one-instanton contribution. In the presence of $N_{f}$ flavors this is given by

$$
\begin{aligned}
I_{1, \mathrm{red}}\left[\kappa^{\prime}, \zeta^{\prime}, N_{f}\right] & =\frac{a^{\zeta^{\prime}}}{2 \pi i} \oint z^{\kappa^{\prime}} I_{1}^{A} I_{1}^{X} I_{1}^{Y} I_{1}^{\xi_{R}} \\
& =\sum_{m=0}^{N_{f}}(-1)^{m} \chi_{[m]}[f] I_{1, \mathrm{red}}\left[\kappa^{\prime}+N_{f} / 2-m, \zeta^{\prime}, N_{f}=0\right],
\end{aligned}
$$

where the $N_{f}=0$ one-instanton contribution on the r.h.s. is evaluated using (4.3) with $\kappa^{\prime}$ replaced by $\kappa^{\prime}+N_{f} / 2-m$.

\subsection{A consistency check}

For $\mathrm{SU}(3)$ the antisymmetric representation is equivalent to the fundamental representation, or more precisely to the antifundamental representation, so we can check our proposal in (4.2) against the instanton partition function for SU(3) with two fundamentals. For simplicity we set $\kappa=\zeta=0$. Let us compare the two instanton partition functions at instanton number $k=1$. Expanding to order $q$ we get

$$
Z_{\text {inst }}=\frac{Z_{\mathrm{QM}}}{Z_{\text {extra }}}=1+\left(I_{1}-I_{1, \text { red }}\right) q+\mathcal{O}\left(q^{2}\right)
$$

where $I_{1}$ is the $k=1$ contribution to $Z_{\mathrm{QM}}$, and $I_{1 \text {,red }}$ is the $k=1$ contribution to $Z_{\mathrm{QM}}$,red . For $\mathrm{SU}(3)+2 \square$ we have ${ }^{5}$

$$
\begin{aligned}
I_{1}^{\mathrm{SU}(3)+2 \square} & =-\frac{1}{2 \pi i} \oint I_{1}^{A} I_{1}^{X} I_{1}^{q} I_{1}^{\xi_{R}} \\
& =\frac{f_{1}+f_{2}}{\sqrt{f_{1} f_{2}}} t^{3}+\left(\frac{f_{1}+f_{2}}{\sqrt{f_{1} f_{2}}} \chi_{\mathbf{2}}[u]-\sqrt{f_{1} f_{2}} \chi_{\mathbf{3}}\left[s_{i}\right]+\frac{1}{\sqrt{f_{1} f_{2}}} \chi_{\overline{\mathbf{3}}}\left[s_{i}\right]\right) t^{4}+\mathcal{O}\left(t^{5}\right),
\end{aligned}
$$

where $f_{1,2}$ are the two flavor fugacities, and $s_{i}(i=1,2,3)$ are the gauge fugacities constrained by $\prod_{i} s_{i}=1$. The counterpart in the reduced QM vanishes in this case,

$$
I_{1, \text { red }}^{\mathrm{SU}(3)+2 \square}=-\frac{1}{2 \pi i} \oint I_{1}^{A} I_{1}^{X} I_{1}^{\xi_{R}}=0,
$$

\footnotetext{
${ }^{5}$ For $\mathrm{SU}(N)+N_{f} k$-instantons there is an overall sign $(-1)^{k\left(\kappa+N_{f} / 2\right)}[23]$.
} 
since there are no contributing poles, as in the $\mathcal{N}=2 \mathrm{U}(N)$ theory [10]. For $\mathrm{SU}(3)+2$ 曰 we get

$$
I_{1}^{\mathrm{SU}(3)+2} \boxminus=\frac{1}{2 \pi i} \oint I_{1}^{A} I_{1}^{X} I_{1}^{q} I_{1}^{Y} I_{1}^{\psi_{R}}=\left(-a \chi_{\mathbf{3}}\left[s_{i}\right]+\frac{1}{a} \chi_{\overline{\mathbf{3}}}\left[s_{i}\right]\right) t^{4}+\mathcal{O}\left(t^{5}\right),
$$

and, using $\kappa^{\prime}=2 \zeta^{\prime}= \pm 2$,

$$
I_{1, \text { red }}^{\mathrm{SU}(3)+2} \boxminus=-t \chi_{\mathbf{2}}[v] Z_{\mathbb{C}^{2} \times \mathbb{C}^{2} / \mathbb{Z}_{2}}=-\chi_{\mathbf{2}}[v]\left(t^{3}+\chi_{\mathbf{2}}[u] t^{4}+\mathcal{O}\left(t^{5}\right)\right) .
$$

Comparing with (4.10) we see that the two instanton partition functions agree if we identify $a=1 / \sqrt{f_{1} f_{2}}$ and $v=\sqrt{f_{1} / f_{2}}$. Note that the agreement holds in this case for both choices of sign in $\Delta \kappa$ and $\Delta \zeta$. However for more general values of $\kappa$ and $\zeta$ the two computations agree only for the lower sign, namely $\kappa^{\prime}=\kappa-2$ and $\zeta^{\prime}=\zeta-1$.

We can further compare the two points of view with an additional flavor. Take $\kappa=\frac{1}{2}$ and $\zeta=0$. For $\mathrm{SU}(3)+3 \square$ we get

$$
\begin{aligned}
I_{1}^{\mathrm{SU}(3)+3 \square}= & -\left(\frac{f_{1}+f_{2}+f_{3}}{\sqrt{f_{1} f_{2} f_{3}}}\right) t^{3} \\
& +\frac{1}{\sqrt{f_{1} f_{2} f_{3}}}\left(\left(f_{1} f_{2}+f_{1} f_{3}+f_{2} f_{3}\right) \chi_{\mathbf{3}}\left[s_{i}\right]+\chi_{\overline{\mathbf{3}}}\left[s_{i}\right]-\left(f_{1}+f_{2}+f_{3}\right) \chi_{\mathbf{2}}[u]\right) t^{4} \\
& +\mathcal{O}\left(t^{5}\right),
\end{aligned}
$$

and as before $I_{1, \text { red }}^{\mathrm{SU}(3)+3} \square=0$. On the other hand for $\mathrm{SU}(3)+2 \boxminus+\square$ we find

$$
\begin{aligned}
I_{1}^{\mathrm{SU}(3)+2} \square+\square= & \frac{1}{2 \pi i} \oint z^{1 / 2} I_{1}^{A} I_{1}^{X} I_{1}^{q} I_{1}^{Y} I_{1}^{\psi_{R}} I_{1}^{\xi_{R}} \\
= & -a \sqrt{f_{3}} t^{3}+\left(\left(\sqrt{f_{3}} \chi_{\mathbf{2}}[v]+\frac{1}{a \sqrt{f_{3}}}\right) \chi_{\mathbf{3}}\left[s_{i}\right]+\frac{a}{\sqrt{f_{3}}} \chi_{\overline{\mathbf{3}}}\left[s_{i}\right]-a \sqrt{f_{3}} \chi_{\mathbf{2}}[u]\right) t^{4} \\
& +\mathcal{O}\left(t^{5}\right)
\end{aligned}
$$

where we denote by $f_{3}$ the fugacity of the single flavor, and, with $\kappa^{\prime}=-\frac{3}{2}$ and $\zeta^{\prime}=-1$,

$$
I_{1, \text { red }}^{\mathrm{SU}(3)+2 \square+\square}=\frac{t\left(v+\frac{1}{v}\right)}{\sqrt{f_{3}}} Z_{\mathbb{C}^{2} \times \mathbb{C}^{2} / \mathbb{Z}_{2}}=\frac{\chi_{\mathbf{2}}[v]}{\sqrt{f_{3}}}\left(t^{3}+\chi_{\mathbf{2}}[u] t^{4}+\mathcal{O}\left(t^{5}\right)\right) .
$$

The two instanton partition functions again agree once we identify $a=1 / \sqrt{f_{1} f_{2}}$ and $v=\sqrt{f_{1} / f_{2}}$.

\section{$5 \quad$ The $\operatorname{Sp}\left(N_{1}\right) \times \operatorname{Sp}\left(N_{2}\right)+(\square, \square)$ theory}

This is the theory obtained from the $\mathbb{Z}_{2}$ orbifold with vector structure of the $\operatorname{Sp}(N)+\square$ theory. Figure 5 shows the structure of the corresponding $1 \mathrm{~d} \mathcal{N}=(0,4)$ gauge theory for $k_{1}$ instantons of $\operatorname{Sp}\left(N_{1}\right)$ and $k_{2}$ instantons of $\operatorname{Sp}\left(N_{2}\right)$. The $1 \mathrm{~d}$ theory admits two discrete theta parameters $\theta_{1}, \theta_{2}$, that are inhereted from the $5 \mathrm{~d}$ theory. The reduced theory is obtained by integrating out the multiplets $q^{(i)}$ and $\psi_{R}^{(i)}$. The Coulomb branch is given by two copies of the expressions in (3.4) and (3.5) for $\varphi_{C}^{(1,2)}$ and $X_{C}^{(1,2)}$ in the orthogonal 


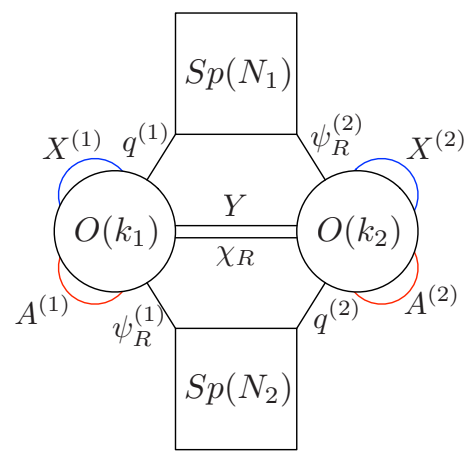

Figure 5. $1 \mathrm{~d} \mathcal{N}=(0,4)$ theory for the $5 \mathrm{~d} \operatorname{Sp}\left(N_{1}\right) \times \operatorname{Sp}\left(N_{2}\right)$ with a bi-fundamental hypermultiplet. The blue and red lines denote symmetric and antisymmetric representations, respectively.

group cases, and $Y_{C}=0$. There is also a twisted Higgs branch on which $\varphi_{T H}^{(1,2)}=0$ and (we assume that $k_{1} \leq k_{2}$ )

$$
\begin{aligned}
X_{T H}^{(1)} & =\operatorname{diag}\left[x_{1}, \ldots, x_{k_{1}}\right] \\
X_{T H}^{(2)} & =\operatorname{diag}\left[x_{1}, \ldots, x_{k_{1}}, x_{k_{1}+1}, \ldots, x_{k_{2}}\right] \\
Y_{T H} & =\operatorname{diag}\left[y_{1}, \ldots, y_{k_{1}}\right] .
\end{aligned}
$$

On the other hand the Higgs branch of the reduced theory is given by $\varphi_{H}^{(1,2)}=Y_{H}=0$ and $X_{H}^{(1,2)}=\operatorname{diag}\left[x_{1}^{(1,2)}, \ldots, x_{k_{1,2}}^{(1,2)}\right]$, where $x_{a}^{(1)}$ and $x_{a}^{(2)}$ are independent. This is a separate branch, and is a remnant of the Higgs branch of the original theory. It corresponds to fractional D0-branes confined to the orbifold fixed plane. We therefore need to divide by the appropriate correction factor $\operatorname{PE}\left[f_{D 0_{c}}\left(q_{1}, q_{2}\right)\right]$. We also need to take into account the shifted discrete theta parameters. The fermion fields $\psi_{L}^{(i)}$ and $\psi_{R}^{(i)}$ will give $N_{1}+N_{2}$ positive mass fermions and $N_{1}+N_{2}$ negative mass fermions charged under $\mathrm{O}\left(k_{i}\right)$, and therefore $\theta_{i}^{\prime}=\theta_{i}+\Delta \theta_{i}$, with

$$
\Delta \theta_{i}=\pi\left[\left(N_{1}+N_{2}\right) \bmod 2\right] .
$$

Our proposal in this case therefore takes the form

$$
Z_{\text {extra }}\left[\theta_{1}, \theta_{2}\right]=\frac{Z_{\mathrm{QM}, \mathrm{red}}\left[\theta_{1}^{\prime}, \theta_{2}^{\prime}\right]}{\operatorname{PE}\left[f_{D 0_{c}}\left(q_{1}, q_{2}\right)\right]} .
$$

The multiparticle index of the reduced theory is given in general by

$$
Z_{\mathrm{QM}, \mathrm{red}}=1+I_{1,0}^{\mathrm{red}} q_{1}+I_{0,1}^{\mathrm{red}} q_{2}+I_{1,1}^{\mathrm{red}} q_{1} q_{2}+I_{2,0}^{\mathrm{red}} q_{1}^{2}+I_{0,2}^{\mathrm{red}} q_{2}^{2}+\mathcal{O}\left(q^{3}\right),
$$

where $I_{k_{1}, k_{2}}^{\mathrm{red}}$ is the index of the $\mathrm{O}\left(k_{1}\right) \times \mathrm{O}\left(k_{2}\right)$ theory. First, we would like to claim that this plethystically-exponentiates to

$$
Z_{\mathrm{QM}, \text { red }}=\operatorname{PE}\left[I_{1,0}^{\mathrm{red}} q_{1}+I_{0,1}^{\mathrm{red}} q_{2}+\left(I_{1,1}^{\mathrm{red}}-I_{1,0}^{\mathrm{red}} I_{0,1}^{\mathrm{red}}\right) q_{1} q_{2}\right] .
$$

We will test this claim shortly by computing some higher order terms. Bust first notice that the first and second terms in the plethystic exponent are the contributions of the 
fractional D0-branes, and the third term is the contribution of the bulk D0-brane. The former are precisely cancelled by the denominator factor leaving

$$
Z_{\text {extra }}=\operatorname{PE}\left[\left(I_{1,1}^{\text {red }}-I_{1,0}^{\text {red }} I_{0,1}^{\text {red }}\right) q_{1} q_{2}\right] .
$$

Now let us compute. The $(1,0)$ contribution is given by

$$
I_{1,0}^{\mathrm{red}}=\frac{1}{2}\left(1+e^{i \theta_{1}^{\prime}}\right) I_{1}^{X}=\frac{1}{2}\left(1+e^{i \theta_{1}^{\prime}}\right) Z_{\mathbb{C}^{2}},
$$

and the $(0,1)$ contribution $I_{0,1}^{\text {red }}$ is similarly given by replacing $\theta_{1}^{\prime}$ by $\theta_{2}^{\prime}$. The $(1,1)$ contribution is given by

$$
\begin{aligned}
I_{1,1}^{\mathrm{red}} & =\frac{1}{4}\left(I_{1}^{X}\right)^{2}\left[I_{1,1}^{Y++} I_{1,1}^{\chi++}+e^{i \theta_{1}^{\prime}} I_{1,1}^{Y-+} I_{1,1}^{\chi-+}+e^{i \theta_{2}^{\prime}} I_{1,1}^{Y+-} I_{1,1}^{\chi+-}+e^{i\left(\theta_{1}^{\prime}+\theta_{2}^{\prime}\right)} I_{1,1}^{Y--} I_{1,1}^{\chi--}\right] \\
& =\frac{t^{3}}{4(1-t u)^{2}\left(1-\frac{t}{u}\right)^{2}}\left[\left(1+e^{i\left(\theta_{1}^{\prime}+\theta_{2}^{\prime}\right)}\right) \frac{1-v^{ \pm 1} u}{1-v^{ \pm 1} t}+\left(e^{i \theta_{1}^{\prime}}+e^{i \theta_{2}^{\prime}}\right) \frac{1+v^{ \pm 1} u}{1+v^{ \pm 1} t}\right]
\end{aligned}
$$

Putting these together, our claim in (5.7) becomes

$$
Z_{\mathrm{QM}, \text { red }}= \begin{cases}\operatorname{PE}\left[\left(q_{1}+q_{2}\right) Z_{\mathbb{C}^{2}}-\left(1+t^{2}\right) q_{1} q_{2} Z_{\mathbb{C}^{2} \times \mathbb{C}^{2} / \mathbb{Z}_{2}}\right] & \left(\theta_{1}^{\prime}, \theta_{2}^{\prime}\right)=(0,0) \\ \operatorname{PE}\left[-t \chi_{2}[v] q_{1} q_{2} Z_{\mathbb{C}^{2} \times \mathbb{C}^{2} / \mathbb{Z}_{2}}\right] & \left(\theta_{1}^{\prime}, \theta_{2}^{\prime}\right)=(\pi, \pi) \\ \operatorname{PE}\left[q_{1} Z_{\mathbb{C}^{2}}\right] & \left(\theta_{1}^{\prime}, \theta_{2}^{\prime}\right)=(0, \pi) \\ \operatorname{PE}\left[q_{2} Z_{\mathbb{C}^{2}}\right] & \left(\theta_{1}^{\prime}, \theta_{2}^{\prime}\right)=(\pi, 0),\end{cases}
$$

where $Z_{\mathbb{C}^{2} \times \mathbb{C}^{2} / \mathbb{Z}_{2}}$ was defined in (4.4), and

$$
Z_{\mathbb{C}^{2}} \equiv \frac{t}{(1-t u)\left(1-\frac{t}{u}\right)} .
$$

To test this claim let us compute a couple of higher order terms. The $(2,0)$ term should be given by

$$
\begin{aligned}
I_{2,0}^{\mathrm{red}} & =\frac{1}{2}\left[\oint I_{2}^{A+} I_{2}^{X+}+e^{i \theta_{1}^{\prime}} I_{2}^{A-} I_{2}^{X-}\right] \\
& = \begin{cases}\frac{t^{2}\left(1+t^{2}\right)}{(1+t u)\left(1+\frac{t}{u}\right)(1-t u)^{2}\left(1-\frac{t}{u}\right)^{2}} & \left(\theta_{1}^{\prime}, \theta_{2}^{\prime}\right)=(0,0),(0, \pi) \\
0 & \left(\theta_{1}^{\prime}, \theta_{2}^{\prime}\right)=(\pi, 0),(\pi, \pi),\end{cases}
\end{aligned}
$$

which agrees with the $q_{1}^{2}$ term in the expansion of (5.11). A similar conclusion holds for the $(0,2)$ term. The $(2,1)$ term should be given by

$$
\begin{aligned}
I_{2,1}^{\mathrm{red}}= & \frac{1}{4} I_{1}^{X}\left[\oint I_{2}^{A+} I_{2}^{X+}\left(I_{2,1}^{Y++} I_{2,1}^{\chi_{R}++}+e^{i \theta_{2}^{\prime}} I_{2,1}^{Y+-} I_{2,1}^{\chi_{R}+-}\right)\right. \\
& \left.+e^{i \theta_{1}^{\prime}} I_{2}^{A-} I_{2}^{X-}\left(I_{2,1}^{Y-+} I_{2,1}^{\chi_{R}-+}+e^{i \theta_{2}^{\prime}} I_{2,1}^{Y--} I_{2,1}^{\chi_{R}--}\right)\right] \\
= & \begin{cases}\frac{\left(t+\frac{1}{t}\right)\left(v^{2}+\frac{1}{v^{2}}-u^{2}-\frac{1}{u^{2}}\right)}{\left(t+\frac{1}{t}-u-\frac{1}{u}\right)^{3}\left(t+\frac{1}{t}+u+\frac{1}{u}\right)\left(v^{2}+\frac{1}{v^{2}}-t^{2}-\frac{1}{t^{2}}\right)} & \left(\theta_{1}^{\prime}, \theta_{2}^{\prime}\right)=(0,0) \\
0 & \left(\theta_{1}^{\prime}, \theta_{2}^{\prime}\right)=(\pi, 0),(0, \pi),(\pi, \pi),\end{cases}
\end{aligned}
$$

which agrees with the $q_{1}^{2} q_{2}$ term. A similar conclusion holds for the $(1,2)$ term. 
Going back to $Z_{\mathrm{QM} \text {,red, }}$, we factor out the contribution of the fractional D0-branes given by $\mathrm{PE}\left[I_{1,0}^{\text {red }} q_{1}+I_{0,1}^{\text {red }} q_{2}\right]$, resulting with

$$
Z_{\text {extra }}= \begin{cases}\operatorname{PE}\left[-\left(1+t^{2}\right) q_{1} q_{2} Z_{\mathbb{C}^{2} \times \mathbb{C}^{2} / \mathbb{Z}_{2}}\right] & \left(\theta_{1}^{\prime}, \theta_{2}^{\prime}\right)=(0,0) \\ \operatorname{PE}\left[-t \chi_{\mathbf{2}}[v] q_{1} q_{2} Z_{\mathbb{C}^{2} \times \mathbb{C}^{2} / \mathbb{Z}_{2}}\right] & \left(\theta_{1}^{\prime}, \theta_{2}^{\prime}\right)=(\pi, \pi) \\ 1 & \left(\theta_{1}^{\prime}, \theta_{2}^{\prime}\right)=(\pi, 0),(0, \pi) .\end{cases}
$$

\subsection{A consistency check}

Since $\operatorname{Sp}(1) \sim \mathrm{SU}(2)$, we can check our proposal (5.15) for the case of $\operatorname{Sp}(1) \times \operatorname{Sp}(1)$ against the instanton partition function obtained in the so-called "SU $(N)$ formalism" by treating each $\mathrm{SU}(2)$ as $\mathrm{U}(2) / \mathrm{U}(1)$ [23] (see also appendix B). Expanding to order $q^{2}$, our proposal gives

$$
\begin{aligned}
Z_{\text {inst }} & =\frac{Z_{\mathrm{QM}}}{Z_{\text {extra }}} \\
& =1+I_{1,0} q_{1}+I_{0,1} q_{2}+I_{2,0} q_{1}^{2}+I_{0,2} q_{2}^{2}+\left(I_{1,1}-I_{1,1}^{\mathrm{red}}+I_{1,0}^{\mathrm{red}} I_{0,1}^{\mathrm{red}}\right) q_{1} q_{2}+\mathcal{O}\left(q^{3}\right)
\end{aligned}
$$

The $(1,0)$ instanton term is given by

$$
\begin{aligned}
I_{1,0}^{\mathrm{inst}} & =I_{1,0} \\
& =\frac{1}{2}\left[I_{1}^{X+} I_{1}^{q+} I_{1}^{\psi_{R}+}+e^{i \theta_{1}} I_{1}^{X-} I_{1}^{q-} I_{1}^{\psi_{R}-}\right] \\
& =\frac{1}{2} \frac{1}{t+\frac{1}{t}-u-\frac{1}{u}}\left(\frac{v+\frac{1}{v}+s+\frac{1}{s}}{t+\frac{1}{t}+s+\frac{1}{s}}+e^{i \theta_{1}} \frac{v+\frac{1}{v}-s-\frac{1}{s}}{t+\frac{1}{t}-s-\frac{1}{s}}\right),
\end{aligned}
$$

which agrees with the corresponding quantity computed in the $\mathrm{SU}(N)$ formalism in [23]. The $(0,1)$ instanton term $I_{0,1}$ likewise agrees. The $(2,0)$ instanton term is given by

$$
\begin{aligned}
I_{2,0}^{\text {inst }} & =I_{2,0} \\
& =\frac{1}{2}\left(\oint I_{2}^{A+} I_{2}^{X+} I_{2}^{q+} I_{2}^{\psi_{R}+}+e^{i \theta_{1}} I_{2}^{A-} I_{2}^{X-} I_{2}^{q-} I_{2}^{\psi_{R}-}\right) \\
& =\left\{\begin{array}{l}
\chi_{\mathbf{3}}[v] t^{4}+\chi_{\mathbf{2}}[v]\left(\chi_{\mathbf{2}}[u] \chi_{\mathbf{2}}[v]-\chi_{\mathbf{2}}\left[s^{\prime}\right] \chi_{\mathbf{2}}[s]\right) t^{5}+\mathcal{O}\left(t^{6}\right) \quad \theta_{1}=0 \\
\chi_{\mathbf{3}}\left[s^{\prime}\right] t^{4}+\chi_{\mathbf{2}}\left[s^{\prime}\right]\left(\chi_{\mathbf{2}}[u] \chi_{\mathbf{2}}\left[s^{\prime}\right]-\chi_{\mathbf{2}}[v] \chi_{\mathbf{2}}[s]\right) t^{5}+\mathcal{O}\left(t^{6}\right) \theta_{1}=\pi
\end{array}\right.
\end{aligned}
$$

where $s, s^{\prime}$ are the fugacities associated to the first and second $\operatorname{Sp}(1)$ factors, respectively. This also agrees with the corresponding quantity computed in the $\mathrm{SU}(N)$ formalism in [23]. The $(0,2)$ instanton term $I_{0,2}$ likewise agrees. The first term where the correction factor $(5.15)$ is relevant is the $(1,1)$ instanton term, given by

$$
\begin{aligned}
I_{1,1}^{\mathrm{inst}} & =I_{1,1}-I_{1,1}^{\mathrm{red}}+I_{1,0}^{\mathrm{red}} I_{0,1}^{\mathrm{red}} \\
& =I_{1,1}-Z_{\mathbb{C}^{2} \times \mathbb{C}^{2} / \mathbb{Z}_{2}} \times \begin{cases}-\left(1+t^{2}\right) & \left(\theta_{1}, \theta_{2}\right)=(0,0) \\
-t \chi_{\mathbf{2}}[v] & \left(\theta_{1}, \theta_{2}\right)=(\pi, \pi) \\
0 & \left(\theta_{1}, \theta_{2}\right)=(\pi, 0),(0, \pi)\end{cases}
\end{aligned}
$$


where

$$
\begin{aligned}
I_{1,1}= & \frac{1}{4}\left[I_{1}^{X}\right]^{2}\left(I_{1,1}^{Y++} I_{1,1}^{\chi_{R}++}\left[I_{1}^{q+}\right]^{2}\left[I_{1}^{\psi_{R}+}\right]^{2}+e^{i \theta_{1}} I_{1,1}^{Y+-} I_{1,1}^{\chi_{R}+-} I_{1}^{q+} I_{1}^{q-} I_{1}^{\psi_{R}+} I_{1}^{\psi_{R}-}\right. \\
& \left.+e^{i \theta_{2}} I_{1,1}^{Y-+} I_{1,1}^{\chi_{R}-+} I_{1}^{q+} I_{1}^{q-} I_{1}^{\psi_{R}+} I_{1}^{\psi_{R^{-}}}+e^{i\left(\theta_{1}+\theta_{2}\right)} I_{1,1}^{Y--} I_{1,1}^{\chi_{R}--}\left[I_{1}^{q-}\right]^{2}\left[I_{1}^{\psi_{R^{-}}}\right]^{2}\right)
\end{aligned}
$$

resulting with

$$
I_{1,1}^{\text {inst }}=\left\{\begin{array}{rr}
t^{2}+\chi_{\mathbf{2}}[u] t^{3}+\chi_{\mathbf{3}}[u] \chi_{\mathbf{3}}[v] t^{4}+ \\
\quad\left(\chi_{\mathbf{2}}[u]\left(\chi_{\mathbf{3}}[u]+2 \chi_{\mathbf{3}}[v]\right)-\chi_{\mathbf{2}}[s] \chi_{\mathbf{2}}\left[s^{\prime}\right] \chi_{\mathbf{2}}[v]\right) t^{5}+\mathcal{O}\left(t^{6}\right) & \left(\theta_{1}, \theta_{2}\right)=(0,0) \\
\chi_{\mathbf{2}}[v] t^{3}+\chi_{\mathbf{2}}[u] \chi_{\mathbf{2}}[v] t^{4}+ & \left(\chi_{\mathbf{2}}[s] \chi_{\mathbf{2}}\left[s^{\prime}\right] \chi_{\mathbf{2}}[u]+\left(\chi_{\mathbf{3}}[u]-1\right) \chi_{\mathbf{2}}[v]\right) t^{5}+\mathcal{O}\left(t^{6}\right) \\
& \left(\theta_{1}, \theta_{2}\right)=(\pi, \pi) \\
\chi_{\mathbf{2}}[v]\left(\chi_{\mathbf{2}}[u] \chi_{\mathbf{2}}\left[s^{\prime}\right]+\chi_{\mathbf{2}}[s] \chi_{\mathbf{2}}[v]\right) t^{5}+\mathcal{O}\left(t^{6}\right) & \left(\theta_{1}, \theta_{2}\right)=(0, \pi) \\
\chi_{\mathbf{2}}[v]\left(\chi_{\mathbf{2}}[u] \chi_{\mathbf{2}}[s]+\chi_{\mathbf{2}}\left[s^{\prime}\right] \chi_{\mathbf{2}}[v]\right) t^{5}+\mathcal{O}\left(t^{6}\right) & \left(\theta_{1}, \theta_{2}\right)=(\pi, 0)
\end{array}\right.
$$

which agrees with the $\mathrm{SU}(N)$ formalism computation in [23]. Note that in this case $\theta_{1,2}^{\prime}=\theta_{1,2}$.

\subsection{Another consistency check}

As one more consistency check we will consider the $(1,1)$ instanton sector of the $\operatorname{Sp}(1) \times$ $\mathrm{Sp}(2)$ theory, where we can compare our result to the result obtained by working in the $\mathrm{SU}(N)$ formalism for the $\mathrm{Sp}(1)=\mathrm{SU}(2)$ factor. This is the simplest instance where both aspects of our proposal are relevant. One has to account for the shift in the discrete theta parameters, as well as for the contribution of the fractional D0-branes. The resulting $(1,1)$ instanton term is given by

$$
\begin{aligned}
I_{1,1}^{\mathrm{inst}}= & I_{1,1}\left[\theta_{1}, \theta_{2}\right]-I_{1,1}^{\mathrm{red}}\left[\theta_{1}+\pi, \theta_{2}+\pi\right]+I_{1,0}^{\mathrm{red}}\left[\theta_{1}+\pi, \theta_{2}+\pi\right] I_{0,1}^{\mathrm{red}}\left[\theta_{1}+\pi, \theta_{2}+\pi\right] \\
= & \left\{\begin{array}{c}
\chi_{\mathbf{2}}[v] t^{3}+\chi_{\mathbf{2}}[v] \chi_{\mathbf{2}}[u] t^{4}+ \\
\chi_{\mathbf{2}}[v]\left(\chi_{\mathbf{3}}[v]+\chi_{\mathbf{3}}[u]-1\right) t^{5}+\mathcal{O}\left(t^{6}\right) \\
\left.t^{2}+\chi_{\mathbf{2}}[u] t_{1}, \theta_{2}\right)=(0,0) \\
\left.\chi_{\mathbf{2}}[u]\left(\chi_{\mathbf{3}}[u]+\chi_{\mathbf{3}}[u]+\chi_{\mathbf{3}}[v]\right) t^{4}+1\right) t^{5}+\mathcal{O}\left(t^{6}\right)\left(\theta_{1}, \theta_{2}\right)=(\pi, \pi) \\
\mathcal{O}\left(t^{6}\right) \quad\left(\theta_{1}, \theta_{2}\right)=(0, \pi),(\pi, 0)
\end{array}\right.
\end{aligned}
$$

This agrees with the result of the $\mathrm{SU}(N)$ formalism (see appendix $\mathrm{B}$ ).

\subsection{Adding flavors}

Adding flavors to the $5 \mathrm{~d}$ gauge theory corresponds to adding Fermi multiplets $\xi_{R}$ to the $1 \mathrm{~d}$ gauge theory as shown in figure 6 . These endow the states with spinor charges under the global $\mathrm{O}\left(2 N_{f_{1}}\right)$ and $\mathrm{O}\left(2 N_{f_{2}}\right)$ symmetries. There are two Weyl spinors that we denote $\mathbf{S}$ and $\mathbf{S}^{\prime}$. Since under the parity element of the $\mathrm{O}(k)$ gauge symmetry $\mathbf{S}$ is even and $\mathbf{S}^{\prime}$ 


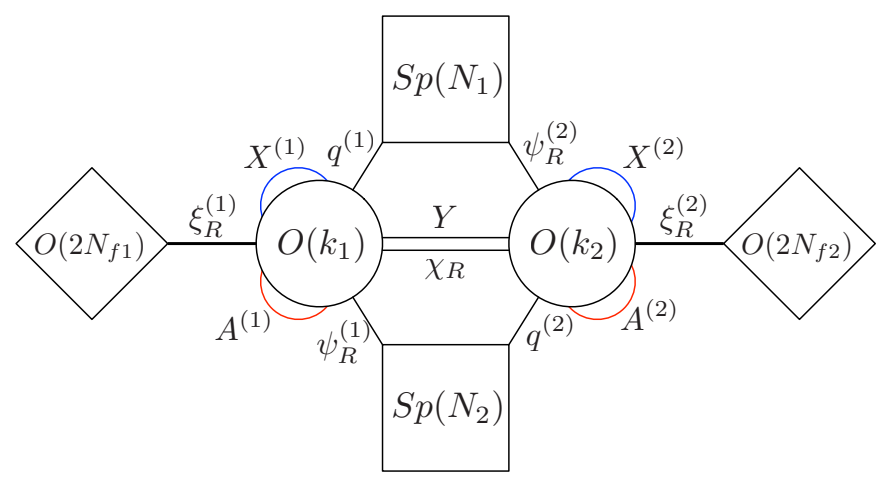

Figure 6. $1 \mathrm{~d} \mathcal{N}=(0,4)$ theory for the $5 \mathrm{~d} \operatorname{Sp}\left(N_{1}\right) \times \operatorname{Sp}\left(N_{2}\right)$ with a bi-fundamental hypermultiplet and fundamental hypermultiplets.

is odd, there is an additional sign in the projection in the latter case. For example, the expression for $I_{1,0}^{\text {red }}$ in (5.9) is replaced with

$$
I_{1,0}^{\mathrm{red}}=\frac{1}{2}\left[\left(1+e^{i \theta_{1}^{\prime}}\right) \chi_{\mathbf{S}_{1}}\left[f_{1}\right]+\left(1-e^{i \theta_{1}^{\prime}}\right) \chi_{\mathbf{S}_{1}^{\prime}}\left[f_{1}\right]\right] Z_{\mathbb{C}^{2}}
$$

As before, we expect that for a small number of flavors equation (5.7) continues to hold. In this case

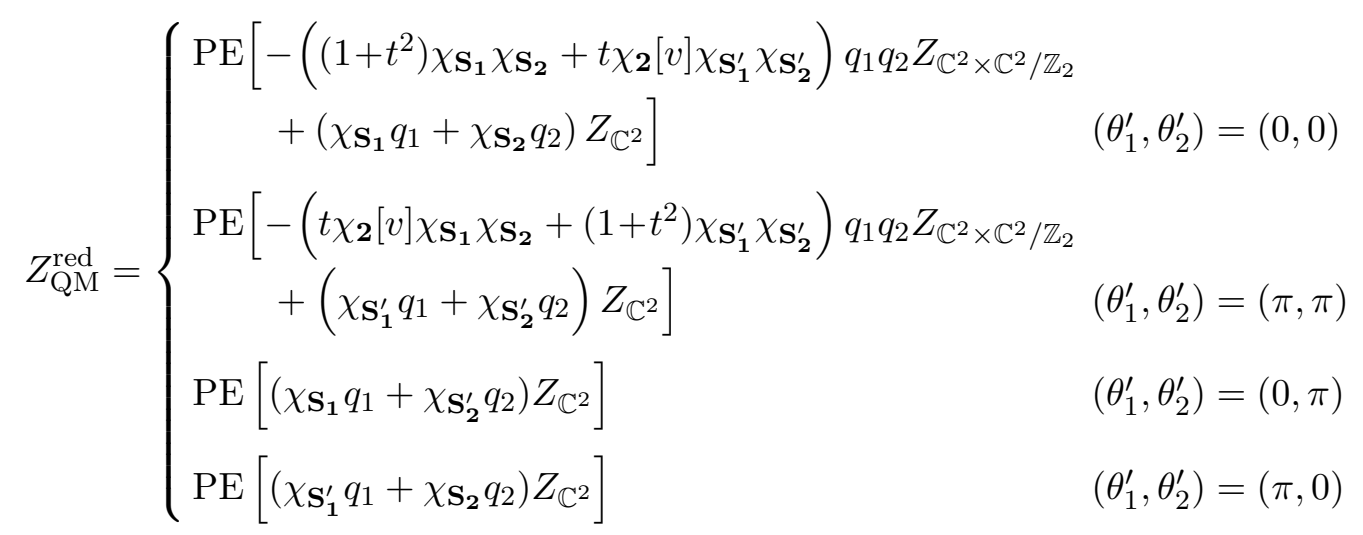

where for brevity we have suppressed the flavor fugacity arguments of the spinor characters. Factoring out the contribution of the fractional D0-branes as before we get

$$
Z_{\text {extra }}= \begin{cases}\operatorname{PE}\left[-\left(\left(1+t^{2}\right) \chi_{\mathbf{S}_{\mathbf{1}}} \chi_{\mathbf{S}_{\mathbf{2}}}+t \chi_{\mathbf{2}}[v] \chi_{\mathbf{S}_{\mathbf{1}}^{\prime}} \chi_{\mathbf{S}_{\mathbf{2}}^{\prime}}\right) q_{1} q_{2} Z_{\mathbb{C}^{2} \times \mathbb{C}^{2} / \mathbb{Z}_{2}}\right] & \left(\theta_{1}^{\prime}, \theta_{2}^{\prime}\right)=(0,0) \\ \mathrm{PE}\left[-\left(t \chi_{\mathbf{2}}[v] \chi_{\mathbf{S}_{1}} \chi_{\mathbf{S}_{\mathbf{2}}}+\left(1+t^{2}\right) \chi_{\mathbf{S}_{\mathbf{1}}^{\prime}} \chi_{\mathbf{S}_{\mathbf{2}}^{\prime}}\right) q_{1} q_{2} Z_{\mathbb{C}^{2} \times \mathbb{C}^{2} / \mathbb{Z}_{2}}\right] & \left(\theta_{1}^{\prime}, \theta_{2}^{\prime}\right)=(\pi, \pi) \\ 1 & \left(\theta_{1}^{\prime}, \theta_{2}^{\prime}\right)=(0, \pi) \\ 1 & \left(\theta_{1}^{\prime}, \theta_{2}^{\prime}\right)=(\pi, 0) .\end{cases}
$$




\section{Conclusions}

In this note we have refined the procedure suggested in [8-10] for obtaining the $5 \mathrm{~d}$ Nekarsov partition function using the $1 \mathrm{~d} \mathcal{N}=(0,4)$ gauge theory constructed from the ADHM data. Our proposal correctly removes the contribution of the extra branches that are generically present when the $5 \mathrm{~d}$ theory has matter in higher representations of the gauge symmetry, and are not associated to the instanton moduli space. In particular it accounts for the possible overcounting in the reduced $1 \mathrm{~d}$ gauge theory in cases where it maintains a remnant of the original Higgs branch, and for the possible shifts in the parameters of the reduced $1 \mathrm{~d}$ theory relative to the original $1 \mathrm{~d}$ theory.

Our proposal is consistent with the results of [10] for the $\mathcal{N}=2$ theories and with the results of [8,9] for the $\mathcal{N}=1 \mathrm{Sp}(N)+\boxminus$ theory. We have applied it also to the theories obtained as $\mathbb{Z}_{2}$ orbifolds of the $\operatorname{Sp}(N)+\square$ theory: the $\mathrm{SU}(N)+2 \boxminus$ theory and the $\operatorname{Sp}\left(N_{1}\right) \times \operatorname{Sp}\left(N_{2}\right)+(\square, \square)$ theory, where no previous results exist, and tested our proposal by exploiting simple isomorphisms between low-rank Lie groups.

An important application of this work is to test the dualities proposed in [23, 24] that involve these theories by comparing the superconformal indices. So far this has only been done in the fractional instanton sectors, where the refinements described in this paper are absent. Our proposal makes it possible to extend the computation to include any $\left(k_{1}, k_{2}\right)$ instanton.

\section{Acknowledgments}

We thank Gabi Zafrir for many useful discussions and participation in early stages of this project. This work is supported in part by the Israel Science Foundation under grant no. 352/13, and by the US-Israel Binational Science Foundation under grant no. 2012-041.

\section{A The $\mathcal{N}=(0,4)$ index}

The $(0,2)$ QM index relevant for the $\mathcal{N}=(0,4)$ theories that we discuss is defined as

$$
I_{k}^{\mathrm{QM}}=\operatorname{Tr}_{k}\left[(-1)^{F} e^{-\beta\left\{Q, Q^{\dagger}\right\}} e^{-2 \epsilon_{+}\left(J_{r}+J_{r^{\prime}}\right)} e^{-2 \epsilon_{-} J_{\ell}} e^{-2 m J_{\ell^{\prime}}} e^{-\alpha_{i} \Pi_{i}} e^{-w_{a} F_{a}}\right],
$$

where the $J$ 's are the generators of the Cartan subgroups of the corresponding $\mathrm{SU}(2)$ symmetries, $\Pi_{i}$ are the generators of the Cartan subgroup of $G_{D 4}$, and $F_{a}$ are the generators of the Cartan subgroup of $G_{D 8}$. The QM Euclidean action is compactified on a circle with circumference $\beta$. The path integral then reduces in the free field limit to Gaussian integrals around zero modes of the gauge field $A_{0}$ and the vector multiplet scalar $\varphi$. The two are combined into a complexified holonomies $\phi=\beta\left(i A_{0}+\varphi\right)$ taking value in the maximal torus of the complexified gauge group $G_{D 0}$,

$$
e^{\phi}= \begin{cases}\operatorname{diag}\left(e^{\phi_{1}}, \ldots, e^{\phi_{k}}\right) & \in \mathrm{U}(k) \\ \operatorname{diag}\left(e^{\sigma_{2} \phi_{1}}, \ldots, e^{\sigma_{2} \phi_{n}}\right) & \in \mathrm{O}(2 n)_{+} \\ \operatorname{diag}\left(e^{\sigma_{2} \phi_{1}}, \ldots, e^{\sigma_{2} \phi_{n-1}}, \sigma_{3}\right) \in \mathrm{O}(2 n)_{-} \\ \operatorname{diag}\left(e^{\sigma_{2} \phi_{1}}, \ldots, e^{\sigma_{2} \phi_{n}}, 1\right) & \in \mathrm{O}(2 n+1)_{+} \\ \operatorname{diag}\left(e^{\sigma_{2} \phi_{1}}, \ldots, e^{\sigma_{2} \phi_{n}},-1\right) & \in \mathrm{O}(2 n+1)_{-} \\ \operatorname{diag}\left(e^{\sigma_{3} \phi_{1}}, \ldots, e^{\sigma_{3} \phi_{k}}\right) & \in \mathrm{Sp}(k)\end{cases}
$$


The result can in general be expressed as a contour integral in $\phi$ of a product of contributions of the different $\mathcal{N}=(0,4)$ multiplets:

$$
I_{k}^{\mathrm{QM}}=\oint_{z_{I}} I_{k}^{A}\left(z_{I}, t\right) I_{k}^{X}\left(z_{I}, t, u\right) I_{k}^{q}\left(z_{I}, t, s_{i}\right) I_{k}^{Y}\left(z_{I}, t, v\right) I_{k}^{\chi_{R}}\left(z_{I}, u, v\right) I_{k}^{\psi_{R}}\left(z_{I}, v, s_{i}\right) I_{k}^{\xi}\left(z_{I}, f_{a}\right),
$$

where following $[9,10]$ we have defined the fugacities

$$
t \equiv e^{-\epsilon_{+}}, u \equiv e^{-\epsilon_{-}}, v \equiv e^{-m}, s_{i} \equiv e^{\alpha_{i}}, f_{a} \equiv e^{-w_{a}}, z_{I} \equiv e^{-\phi_{I}}
$$

The contributions of the different $(0,4)$ multiplets can be determined by decomposing them into $(0,2)$ multiplets, whose contributions have a well known structure $[9,10]$. We will give the explicit expressions for the different theories below. In particular the contribution of the vector multiplet includes the Haar measure $\left[d z_{I}\right]$.

Preforming the integral one must provide a prescription for the contours, namely for how to deal with the poles. This question was answered for $2 \mathrm{~d}$ gauge theories with $\mathcal{N}=$ $(2,2)$ and $\mathcal{N}=(0,2)$ supersymmetry in $[25,26]$ using the Jeffrey-Kirwan (JK) residue rule. The quantity of interest in that case is the elliptic genus, a refinement of the partition function on the torus. Since $\mathcal{N}=(0,4)$ QM may be regarded as the dimensional reduction of a $2 \mathrm{~d} \mathcal{N}=(0,4)$ field theory, which is a special case of a $2 \mathrm{~d} \mathcal{N}=(0,2)$ theory, the same rule can be applied to the QM index, which corresponds to a limit of the $2 \mathrm{~d}$ elliptic genus. This is the approach taken in [9]. The authors of [9] have also noted that the JK prescription is equivalent, at least in the examples they studied, to integrating each of the complex U(1) holonomies separately on a unit circle, and applying the following residue rules: poles arising from the $5 \mathrm{~d}$ gauge multiplet contribute when they are inside the contour, poles arising from $5 \mathrm{~d}$ matter hypermultiplets contribute when they are outside the contour, and poles at the origin do not contribute. The final result is

$$
I_{k}^{\mathrm{QM}}=\frac{1}{\left|W_{G_{D 0}}\right|} \sum \mathrm{JK}-\mathrm{Res}
$$

where $W_{G_{D 0}}$ is the Weyl group of $G_{D 0}$. In particular

$$
\begin{aligned}
& |W|_{\mathrm{U}(k)}=k !, \quad|W|_{\mathrm{O}(2 n)_{+}}=2^{n-1} n !, \quad|W|_{\mathrm{O}(2 n)_{-}}=2^{n-1}(n-1) ! \\
& |W|_{\mathrm{O}(2 n+1)_{+}}=2^{n} n !, \quad|W|_{\mathrm{O}(2 n+1)_{-}}=2^{n} n !, \quad|W|_{\mathrm{Sp}(k)}=k ! .
\end{aligned}
$$

For $G_{D 0}=O$ one must sum over the two holonomy sectors $O_{+}$and $O_{-}$, with a relative weight given by the discrete theta parameter as follows [18]:

$$
I_{k}^{\mathrm{QM}}=\left\{\begin{array}{l}
\frac{1}{2}\left(I_{+}^{k}+I_{-}^{k}\right), \quad \theta=0 \\
\frac{(-1)^{k}}{2}\left(I_{+}^{k}-I_{-}^{k}\right) \theta=\pi
\end{array}\right.
$$

In the remaining part of this appendix we will present the formulas for the contributions of the $(0,4)$ multiplets that arise for the $5 \mathrm{~d} \mathcal{N}=1$ theories featured in this paper. This information can also be extracted from [23], where it is organized by the $5 \mathrm{~d}$ multiplets. For brevity we will adopt the shorthand $1-u^{ \pm 1} t=(1-u t)\left(1-u^{-1} t\right)$, etc. 


\section{A.1 $\operatorname{Sp}(N)+\boxminus$}

The content of the $1 \mathrm{~d}$ theory in this case is shown in figure 2. There are four cases to consider, $\mathrm{O}(k)_{\epsilon}$ with $k=2 n, 2 n+1$ and $\epsilon= \pm$. For $k=2 n+1$ we have:

$$
\begin{aligned}
I_{k}^{A, \epsilon} & =\frac{\epsilon^{n}}{2^{n} n !}\left(\frac{1}{t}-t\right) \prod_{I=1}^{n} \frac{\left(1-\epsilon z_{I}^{ \pm 1} t^{2}\right)\left(z_{I}+\frac{1}{z_{I}}-2 \epsilon\right) d z_{I}}{t^{2} z_{I}} \prod_{I<J}^{n} \frac{\left(1-z_{I}^{ \pm 1} z_{J}^{ \pm 1} t^{2}\right)\left(z_{I}+\frac{1}{z_{I}}-z_{J}-\frac{1}{z_{J}}\right)^{2}}{t^{4}} \\
I_{k}^{X, \epsilon} & =\frac{t^{n+1}}{\left(1-u^{ \pm 1} t\right)^{n+1}} \prod_{I=1}^{n} \frac{t^{4}}{\left(1-\epsilon z_{I}^{ \pm 1} u^{ \pm 1} t\right)\left(1-z_{I}^{ \pm 2} u^{ \pm 1} t\right)} \prod_{I<J}^{n} \frac{t^{4}}{\left(1-z_{I}^{ \pm 1} z_{J}^{ \pm 1} u^{ \pm 1} t\right)} \\
I_{k}^{q, \epsilon} & =\prod_{i=1}^{N} \frac{t}{\left(1-\epsilon s_{i}^{ \pm 1} t\right)} \prod_{I=1}^{n} \prod_{i=1}^{N} \frac{t^{2}}{\left(1-s_{i}^{ \pm 1} z_{I}^{ \pm 1} t\right)} \\
I_{k}^{Y, \epsilon} & =\frac{t^{n+1}}{\left(1-v^{ \pm 1} t\right)^{n+1}} \prod_{I=1}^{n} \frac{t^{4}}{\left(1-\epsilon z_{I}^{ \pm 1} v^{ \pm 1} t\right)\left(1-z_{I}^{ \pm 2} v^{ \pm 1} t\right)} \prod_{I<J}^{n} \frac{t^{4}}{\left(1-z_{I}^{ \pm 1} z_{J}^{ \pm 1} v^{ \pm 1} t\right)} \\
I_{k}^{\psi_{R}, \epsilon} & =\prod_{i=1}^{N} \frac{\left(1-\epsilon s_{i}^{ \pm 1} v\right)}{v} \prod_{I=1}^{n} \prod_{i=1}^{N} \frac{\left(1-s_{i}^{ \pm 1} z_{I}^{ \pm 1} v\right)}{v^{2}} \\
I_{k}^{\chi_{R}, \epsilon} & =\frac{\left(1-v^{ \pm 1} u\right)^{n}}{u^{n}} \prod_{I=1}^{n} \frac{\left(1-\epsilon z_{I}^{ \pm 1} v^{ \pm 1} u\right)}{u^{2}} \prod_{I<J}^{n} \frac{\left(1-z_{I}^{ \pm 1} z_{J}^{ \pm 1} v^{ \pm 1} u\right)}{u^{4}} \\
I_{k}^{\xi_{R}, \epsilon} & =\prod_{a=1}^{N_{f}} \prod_{I=1}^{n}\left(f_{a}+\frac{1}{f_{a}}-z_{I}-\frac{1}{z_{I}}\right) \prod_{a=1}^{N_{f}}\left(\sqrt{f_{a}}-\epsilon \frac{1}{\sqrt{f_{a}}}\right)
\end{aligned}
$$

For $k=2 n$ we have:

$$
\begin{aligned}
I_{k}^{A, \epsilon}= \begin{cases}\frac{\left(\frac{1}{t}-t\right)^{n}}{2^{n-1} n !} \prod_{I=1}^{n} \frac{d z_{I}}{z_{I}} \prod_{I<J}^{n} \frac{\left(1-z_{I}^{ \pm 1} z_{J}^{ \pm 1} t^{2}\right)\left(z_{I}+\frac{1}{z_{I}}-z_{J}-\frac{1}{z_{J}}\right)^{2}}{t^{4}} & \epsilon=+ \\
\frac{(-1)^{n}\left(\frac{1}{t}-t\right)^{n-1}\left(\frac{1}{t}+t\right)}{2^{n-1}(n-1) !} \prod_{I=1}^{n-1} \frac{\left(1-z_{I}^{ \pm 2} t^{4}\right)\left(z_{I}^{2}+\frac{1}{z_{I}^{2}}-2\right) d z_{I}}{t^{4} z_{I}} \prod_{I<J}^{n-1} \frac{\left(1-z_{I}^{ \pm 1} z_{J}^{ \pm 1} t^{2}\right)\left(z_{I}+\frac{1}{z_{I}}-z_{J}-\frac{1}{z_{J}}\right)^{2}}{t^{4}} & \epsilon=-\end{cases} \\
I_{k}^{X, \epsilon}= \begin{cases}\frac{t^{n}}{\left(1-u^{ \pm 1} t\right)^{n}} \prod_{I=1}^{n} \frac{t^{2}}{\left(1-z_{I}^{ \pm 2} u^{ \pm 1} t\right)} \prod_{I<J}^{n} \frac{t^{4}}{\left(1-z_{I}^{ \pm 1} z_{J}^{ \pm 1} u^{ \pm 1} t\right)} \\
\frac{t^{n+2}}{\left(1+u^{ \pm 1} t\right)\left(1-u^{ \pm 1} t\right)^{n+1}} \prod_{I=1}^{n-1} \frac{t^{4}}{\left(1-z_{I}^{ \pm 2} u^{ \pm 1} t\right)\left(1-z_{I}^{ \pm 2} u^{ \pm 1} t\right)} \prod_{I<J}^{n-1} \frac{t^{4}}{\left(1-z_{I}^{ \pm 1} z_{J}^{ \pm 1} u^{ \pm 1} t\right)} \epsilon=-\end{cases} \\
I_{k}^{q, \epsilon}= \begin{cases}\prod_{I=1}^{n} \prod_{i=1}^{N} \frac{t^{2}}{\left(1-s_{i}^{ \pm 1} z_{I}^{ \pm 1} t\right)} \\
\prod_{i=1}^{N} \frac{t^{2}}{\left(1-s_{i}^{ \pm 2} t^{2}\right)} \prod_{I=1}^{n-1} \prod_{i=1}^{N} \frac{t^{2}}{\left(1-s_{i}^{ \pm 1} z_{I}^{ \pm 1} t\right)} \epsilon=-\end{cases}
\end{aligned}
$$




$$
\begin{aligned}
& I_{k}^{Y, \epsilon}= \begin{cases}\frac{t^{n}}{\left(1-v^{ \pm 1} t\right)^{n}} \prod_{I=1}^{n} \frac{t^{2}}{\left(1-z_{I}^{ \pm 2} v^{ \pm 1} t\right)} \prod_{I<J}^{n} \frac{t^{4}}{\left(1-z_{I}^{ \pm 1} z_{J}^{ \pm 1} v^{ \pm 1} t\right)} & \epsilon=+ \\
\frac{t^{n+2}}{\left(1+v^{ \pm 1} t\right)\left(1-v^{ \pm 1} t\right)^{n+1}} \prod_{I=1}^{n-1} \frac{t^{4}}{\left(1-z_{I}^{ \pm 2} v^{ \pm 1} t\right)\left(1-z_{I}^{ \pm 2} v^{ \pm 1} t\right)} \prod_{I<J}^{n-1} \frac{t^{4}}{\left(1-z_{I}^{ \pm 1} z_{J}^{ \pm 1} v^{ \pm 1} t\right)} \epsilon=-\end{cases} \\
& I_{k}^{\psi_{R}, \epsilon}= \begin{cases}\prod_{I=1}^{n} \frac{1}{N} \frac{\left(1-s_{i}^{ \pm 1} z_{I}^{ \pm 1} v\right)}{v^{2}} & \epsilon=+ \\
\prod_{i=1}^{N} \frac{\left(1-s_{i}^{ \pm 2} v^{2}\right)}{v^{2}} \prod_{I=1}^{n-1} \prod_{i=1}^{N} \frac{\left(1-s_{i}^{ \pm 1} z_{I}^{ \pm 1} v\right)}{v^{2}} \epsilon=-\end{cases} \\
& I_{k}^{\chi_{R}, \epsilon}= \begin{cases}\frac{\left(1-v^{ \pm 1} u\right)^{n}}{u^{n}} \prod_{I<J}^{n} \frac{\left(1-z_{I}^{ \pm 1} z_{J}^{ \pm 1} v^{ \pm 1} u\right)}{u^{4}} & \epsilon=+ \\
\frac{\left(1+v^{ \pm 1} u\right)\left(1-v^{ \pm 1} u\right)^{n-1}}{u^{n}} \prod_{I=1}^{n-1} \frac{\left(1-z_{I}^{ \pm 2} v^{ \pm 2} u^{2}\right)}{u^{4}} \prod_{I<J}^{n-1} \frac{\left(1-z_{I}^{ \pm 1} z_{J}^{ \pm 1} v^{ \pm 1} u\right)}{u^{4}} \epsilon=- & \epsilon=-\end{cases} \\
& I_{k}^{\xi_{R}, \epsilon}= \begin{cases}\prod_{a=1}^{N_{f}} \prod_{I=1}^{n}\left(f_{a}+\frac{1}{f_{a}}-z_{I}-\frac{1}{z_{I}}\right) & \epsilon=+ \\
\prod_{a=1}^{N_{f}} \prod_{I=1}^{n-1}\left(f_{a}+\frac{1}{f_{a}}-z_{I}-\frac{1}{z_{I}}\right) \prod_{a=1}^{N_{f}}\left(f_{a}-\frac{1}{f_{a}}\right) \epsilon=-\end{cases}
\end{aligned}
$$

\section{A.2 $\mathrm{SU}(N)+2 \boxminus$}

The content of the $1 \mathrm{~d}$ theory in this case is shown in figure 4 . The contributions of the different $1 \mathrm{~d}(0,4)$ multiplets are given by:

$$
\begin{aligned}
I_{k}^{A} & =\left(\frac{1}{t}-t\right)^{k} \prod_{I=1}^{k} \frac{d z_{I}}{z_{I}} \prod_{I<J}^{k} \frac{\left(1-\left(\frac{z_{I}}{z_{J}}\right)^{ \pm 1}\right)\left(1-\left(\frac{z_{I}}{z_{J}}\right)^{ \pm 1} t^{2}\right)}{t^{2}} \\
I_{k}^{q} & =\prod_{I=1}^{k} \prod_{i=1}^{N} \frac{t}{\left(1-\left(\frac{z_{I}}{s_{i}}\right)^{ \pm 1} t\right)} \\
I_{k}^{X} & =\prod_{I=1}^{k} \frac{t}{\left(1-u^{ \pm 1} t\right)} \prod_{I<J}^{k} \frac{t^{2}}{\left(1-\left(\frac{z_{I}}{z_{J}}\right)^{ \pm 1} u^{ \pm 1} t\right)} \\
I_{k}^{Y} & =\prod_{I=1}^{k} \frac{t^{2}}{\left(1-\left(a z_{I}^{2}\right)^{ \pm 1} v^{ \pm 1} t\right)} \prod_{I<J}^{k} \frac{t^{2}}{\left(1-\left(a z_{I} z_{J}\right)^{ \pm 1} v^{ \pm 1} t\right)} \\
I_{k}^{\psi_{R}} & =\prod_{I=1}^{k} \prod_{i=1}^{N} \frac{\left(1-\left(a z_{I} s_{i}\right)^{ \pm 1} v\right)}{v}
\end{aligned}
$$




$$
\begin{aligned}
I_{k}^{\chi_{R}} & =\prod_{I<J}^{k} \frac{\left(1-\left(a z_{I} z_{J}\right)^{ \pm 1} v^{ \pm 1} u\right)}{u^{2}} \\
I_{k}^{\xi_{R}} & =\prod_{I=1}^{k} \prod_{a=1}^{N_{f}}\left(\sqrt{z_{I} f_{a}}-\frac{1}{\sqrt{z_{I} f_{a}}}\right)
\end{aligned}
$$

We have included here the fugacity $a$ of the additional $\mathrm{U}(1)_{\ell^{\prime}}$ symmetry.

A.3 $\operatorname{Sp}\left(N_{1}\right) \times \operatorname{Sp}\left(N_{2}\right)+(\square, \square)$

The content of the $1 \mathrm{~d}$ theory in this case is shown in figure 6 . The contributions of $A$, $X, q, \psi_{R}$ and $\xi_{R}$ are essentially the same as in the $\operatorname{Sp}(N)+$ 日 theory, with $N$ replaced by either $N_{1}$ or $N_{2}$, and $n$ replaced by either $n_{1}$ or $n_{2}$, as can be read off directly from the quiver diagram. The remaining contributions of $Y$ and $\chi_{R}$ are given for $k_{1}=2 n_{1}$ and $k_{2}=2 n_{2}$ by:

$$
\begin{aligned}
& I_{k_{1}, k_{2}}^{Y, \epsilon_{1}, \epsilon_{2}}=\left\{\begin{array}{lr}
\prod_{I, J=1}^{n_{1}, n_{2}} \frac{t^{4}}{\left(1-z_{1 I}^{ \pm 1} z_{2 J}^{ \pm 1} v^{ \pm 1} t\right)} & \left(\epsilon_{1}, \epsilon_{2}\right)=(+,+) \\
\prod_{I=1}^{n_{1}} \frac{t^{4}}{\left(1-z_{1 I}^{ \pm 2} v^{ \pm 2} t^{2}\right)} \prod_{I, J=1}^{n_{1}, n_{2}-1} \frac{t^{4}}{\left(1-z_{1 I}^{ \pm 1} z_{2 J}^{ \pm 1} v^{ \pm 1} t\right)} & \left(\epsilon_{1}, \epsilon_{2}\right)=(+,-) \\
\prod_{J=1}^{n_{2}} \frac{t^{4}}{\left(1-z_{2 J}^{ \pm 2} v^{ \pm 2} t^{2}\right)} \prod_{I, J=1}^{n_{1}-1, n_{2}} \frac{t^{4}}{\left(1-z_{1 I}^{ \pm 1} z_{2 J}^{ \pm 1} v^{ \pm 1} t\right)} & \left(\epsilon_{1}, \epsilon_{2}\right)=(-,+) \\
\frac{t^{2}}{\left(1-v^{ \pm 2} t^{2}\right)} \prod_{I=1}^{n_{1}-1} \frac{t^{4}}{\left(1-z_{1 I}^{ \pm 2} v^{ \pm 2} t^{2}\right)} \prod_{J=1}^{n_{2}-1} \frac{t^{4}}{\left(1-z_{2 J}^{ \pm 2} v^{ \pm 2} t^{2}\right)} \prod_{I, J=1}^{n_{1}-1, n_{2}-1} \frac{t^{4}}{\left(1-z_{1 I}^{ \pm 1} z_{2 J}^{ \pm 1} v^{ \pm 1} t\right)}\left(\epsilon_{1}, \epsilon_{2}\right)=(-,-)
\end{array}\right. \\
& I_{k_{1}, k_{2}}^{\chi_{R}, \epsilon_{1}, \epsilon_{2}}=\left\{\begin{array}{lr}
\prod_{I, J=1}^{n_{1}, n_{2}} \frac{\left(1-z_{1 I}^{ \pm 1} z_{2,}^{ \pm 1} v^{ \pm 1} u\right)}{u^{4}} & \left(\epsilon_{1}, \epsilon_{2}\right)=(+,+) \\
\prod_{I=1}^{n_{1}} \frac{\left(1-z_{1 I}^{ \pm 2} v^{ \pm 2} u^{2}\right)}{u^{4}} \prod_{I, J=1}^{n_{1}, n_{2}-1} \frac{\left(1-z_{1 I}^{ \pm 1} z_{2 J}^{ \pm 1} v^{ \pm 1} u\right)}{u^{4}} & \left(\epsilon_{1}, \epsilon_{2}\right)=(+,-) \\
\prod_{J=1}^{n_{2}} \frac{\left(1-z_{2 J}^{ \pm 2} v^{ \pm 2} u^{2}\right)}{u^{4}} \prod_{I, J=1}^{n_{1}-1, n_{2}} \frac{\left(1-z_{1 I}^{ \pm 1} z_{2,}^{ \pm 1} v^{ \pm 1} u\right)}{u^{4}} & \left(\epsilon_{1}, \epsilon_{2}\right)=(-,+) \\
\frac{\left(1-v^{ \pm 2} u^{2}\right)}{u^{2}} \prod_{I=1}^{n_{1}-1} \frac{\left(1-z_{1 I}^{ \pm 2} v^{ \pm 2} u^{2}\right)}{u^{4}} \prod_{J=1}^{n_{2}-1} \frac{\left(1-z_{2 J}^{ \pm 2} v^{ \pm 2} u^{2}\right)}{u^{4}} \prod_{I, J=1}^{n_{1}-1, n_{2}-1} \frac{\left(1-z_{1 I}^{ \pm 1} z_{2 J}^{ \pm 1} v^{ \pm 1} u\right)}{u^{4}}\left(\epsilon_{1}, \epsilon_{2}\right)=(-,-) & \left.\prod_{I J}\right)
\end{array}\right.
\end{aligned}
$$

and for $k_{1}=2 n_{1}+1$ and $k_{2}=2 n_{2}+1$ by:

$$
\begin{gathered}
I_{k_{1}, k_{2}}^{Y, \epsilon_{1}, \epsilon_{2}}=\frac{t}{\left(1-\epsilon_{1} \epsilon_{2} v^{ \pm 1} t\right)} \prod_{I=1}^{n_{1}} \frac{t^{2}}{\left(1-\epsilon_{2} z_{1 I}^{ \pm 1} v^{ \pm 1} t\right)} \prod_{J=1}^{n_{2}} \frac{t^{2}}{\left(1-\epsilon_{1} z_{2 J}^{ \pm 1} v^{ \pm 1} t\right)} \prod_{I, J=1}^{n_{1}, n_{2}} \frac{t^{4}}{\left(1-z_{1 I}^{ \pm 1} z_{2 J}^{ \pm 1} v^{ \pm 1} t\right)} \\
I_{k_{1}, k_{2}}^{\chi_{R}, \epsilon_{1}, \epsilon_{2}}=\frac{\left(1-\epsilon_{1} \epsilon_{2} v^{ \pm 1} u\right)}{u} \prod_{I=1}^{n_{1}} \frac{\left(1-\epsilon_{2} z_{1 I}^{ \pm 1} v^{ \pm 1} u\right)}{u^{2}} \prod_{J=1}^{n_{2}} \frac{\left(1-\epsilon_{1} z_{2 J}^{ \pm 1} v^{ \pm 1} u\right)}{u^{2}} \prod_{I, J=1}^{n_{1}, n_{2}} \frac{\left(1-z_{1 I}^{ \pm 1} z_{2 J}^{ \pm 1} v^{ \pm 1} u\right)}{u^{4}},
\end{gathered}
$$


and for $k_{1}=2 n_{1}+1$ and $k_{2}=2 n_{2}$ by:

$$
\begin{aligned}
& I_{k_{1}, k_{2}}^{Y, \epsilon_{1}, \epsilon_{2}}= \begin{cases}\prod_{J=1}^{n_{2}} \frac{t^{2}}{\left(1-\epsilon_{1} z_{2 J}^{ \pm 1} v^{ \pm 1} t\right)} \prod_{I, J=1}^{n_{1}, n_{2}} \frac{t^{4}}{\left(1-z_{1 I}^{ \pm 1} z_{2 J}^{ \pm 1} v^{ \pm 1} t\right)} & \epsilon_{2}=+ \\
\frac{t^{2}}{\left(1-v^{ \pm 2} t^{2}\right)} \prod_{J=1}^{n_{2}-1} \frac{t^{2}}{\left(1-\epsilon_{1} z_{2 J}^{ \pm 1} v^{ \pm 1} t\right)} \prod_{I=1}^{n_{1}} \frac{t^{4}}{\left(1-z_{1 I}^{ \pm 2} v^{ \pm 2} t^{2}\right)} \prod_{I, J=1}^{n_{1}, n_{2}-1} \frac{t^{4}}{\left(1-z_{1 I}^{ \pm 1} z_{2 J}^{ \pm 1} v^{ \pm 1} t\right)} \epsilon_{2}=-\end{cases} \\
& I_{k_{1}, k_{2}}^{\chi_{R}, \epsilon_{1}, \epsilon_{2}}= \begin{cases}\prod_{J=1}^{n_{2}} \frac{\left(1-\epsilon_{1} z_{2 J}^{ \pm 1} v^{ \pm 1} u\right)}{u^{2}} \prod_{I, J=1}^{n_{1}, n_{2}} \frac{\left(1-z_{1 I}^{ \pm 1} z_{2 J}^{ \pm 1} v^{ \pm 1} u\right)}{u^{4}} & \epsilon_{2}=+ \\
\frac{\left(1-v^{ \pm 2} u^{2}\right)}{u^{2}} \prod_{J=1}^{n_{2}-1} \frac{\left(1-\epsilon_{1} z_{2 J}^{ \pm 1} v^{ \pm 1} u\right)}{u^{2}} \prod_{I=1}^{n_{1}} \frac{\left(1-z_{1 I}^{ \pm 2} v^{ \pm 2} u^{2}\right)}{u^{4}} \prod_{I, J=1}^{n_{1}, n_{2}-1} \frac{\left(1-z_{1 I}^{ \pm 1} z_{2 J}^{ \pm 1} v^{ \pm 1} u\right)}{u^{4}} \epsilon_{2}=-\end{cases}
\end{aligned}
$$

The case $k_{1}=2 n_{1}$ and $k_{2}=2 n_{2}+1$ is related to the last one by replacing $n_{1} \leftrightarrow n_{2}$, $z_{1 I} \leftrightarrow z_{2 J}$ and $\epsilon_{1} \leftrightarrow \epsilon_{2}$.

\section{B $\quad \mathrm{SU}(2) \times \mathrm{Sp}(2)$ in the $\mathrm{SU}(N)$ formalism}

The $\mathrm{SU}(N)$ instanton partition function is generically extracted from the $\mathrm{U}(N)$ one by imposing the constraint $\prod_{i=1}^{N} s_{i}=1$. However there are cases where a correction factor must be included to remove the contribution of another type of extraneous states. In 5 -brane constructions these correspond to compact string states that are external to the 5-brane web [27]. The general structure of the factor is

$$
Z_{\text {extra }}=P E\left[\frac{t^{2} \mathcal{F}}{(1-t u)\left(1-\frac{t}{u}\right)}\right]
$$

where $\mathcal{F}$ depends the instanton and global symmetry fugacities, and is determined on a case by case basis, typically by requiring the invariance of the full partition function under $t \rightarrow 1 / t$, which is a part of the conformal symmetry. For $\mathrm{SU}(2) \times \mathrm{Sp}(2)$ we find that $\mathcal{F}=\left(v^{2}+v^{-2}\right) q_{1}$, and therefore

$$
Z^{\mathrm{SU}(2)_{\theta_{1}} \times \operatorname{Sp}(2)_{\theta_{2}}}=P E\left[\frac{t^{2}\left(v^{2}+\frac{1}{v^{2}}\right) q_{1}}{(1-t u)\left(1-\frac{t}{u}\right)}\right] Z^{\mathrm{U}(2)_{\theta_{1} / \pi} \times \operatorname{Sp}(2)_{\theta_{2}} /\left\{s_{1} s_{2}=1\right\}} .
$$

This can now be computed by incorporating the results of [27] for $\mathrm{U}(N) \times \operatorname{Sp}(N)$. We will not do this explicitly here, but rest assured that the result at order $q_{1} q_{2}$ agrees with (5.21).

Open Access. This article is distributed under the terms of the Creative Commons Attribution License (CC-BY 4.0), which permits any use, distribution and reproduction in any medium, provided the original author(s) and source are credited. 


\section{References}

[1] M.F. Atiyah, N.J. Hitchin, V.G. Drinfeld and Yu. I. Manin, Construction of Instantons, Phys. Lett. A 65 (1978) 185 [InSPIRE].

[2] E. Witten, $\sigma$-models and the ADHM construction of instantons, J. Geom. Phys. 15 (1995) 215 [hep-th/9410052] [INSPIRE].

[3] N.A. Nekrasov, Seiberg-Witten prepotential from instanton counting, Adv. Theor. Math. Phys. 7 (2003) 831 [hep-th/0206161] [INSPIRE].

[4] N. Nekrasov and S. Shadchin, ABCD of instantons, Commun. Math. Phys. 252 (2004) 359 [hep-th/0404225] [INSPIRE].

[5] S. Shadchin, On certain aspects of string theory/gauge theory correspondence, Ph.D. Thesis, Orsay LPT, Paris France (2005) [hep-th/0502180] [INSPIRE].

[6] M.R. Douglas, Gauge fields and D-branes, J. Geom. Phys. 28 (1998) 255 [hep-th/9604198] [INSPIRE].

[7] H.-C. Kim, S. Kim, E. Koh, K. Lee and S. Lee, On instantons as Kaluza-Klein modes of M5-branes, JHEP 12 (2011) 031 [arXiv:1110.2175] [INSPIRE].

[8] H.-C. Kim, S.-S. Kim and K. Lee, 5-dim Superconformal Index with Enhanced En Global Symmetry, JHEP 10 (2012) 142 [arXiv:1206.6781] [INSPIRE].

[9] C. Hwang, J. Kim, S. Kim and J. Park, General instanton counting and 5d SCFT, JHEP 07 (2015) 063 [arXiv:1406.6793] [INSPIRE].

[10] Y. Hwang, J. Kim and S. Kim, M5-branes, orientifolds and S-duality, JHEP 12 (2016) 148 [arXiv: 1607.08557] [INSPIRE].

[11] N. Seiberg, Five-dimensional SUSY field theories, nontrivial fixed points and string dynamics, Phys. Lett. B 388 (1996) 753 [hep-th/9608111] [INSPIRE].

[12] O. Bergman and D. Rodriguez-Gomez, $5 d$ quivers and their AdS $S_{6}$ duals, JHEP 07 (2012) 171 [arXiv: 1206.3503] [INSPIRE].

[13] O. Aharony, M. Berkooz, S. Kachru and E. Silverstein, Matrix description of $(1,0)$ theories in six-dimensions, Phys. Lett. B 420 (1998) 55 [hep-th/9709118] [INSPIRE].

[14] D. Tong, The holographic dual of $A d S_{3} \times S^{3} \times S^{3} \times S^{1}$, JHEP 04 (2014) 193 [arXiv: 1402.5135] [INSPIRE].

[15] D. Tong and K. Wong, Instantons, Wilson lines and D-branes, Phys. Rev. D 91 (2015) 026007 [arXiv: 1410 . 8523] [INSPIRE].

[16] Y. Tachikawa, On S-duality of 5d super Yang-Mills on $S^{1}$, JHEP 11 (2011) 123 [arXiv:1110.0531] [INSPIRE].

[17] B. Collie and D. Tong, Instantons, Fermions and Chern-Simons Terms, JHEP 07 (2008) 015 [arXiv: 0804.1772] [INSPIRE].

[18] O. Bergman, D. Rodríguez-Gómez and G. Zafrir, Discrete $\theta$ and the $5 d$ superconformal index, JHEP 01 (2014) 079 [arXiv:1310.2150] [INSPIRE].

[19] A. Keurentjes and S. Sethi, Twisting E8 five-branes, Phys. Rev. D 66 (2002) 046001 [hep-th/0205162] [INSPIRE].

[20] D.R. Morrison and N. Seiberg, Extremal transitions and five-dimensional supersymmetric field theories, Nucl. Phys. B 483 (1997) 229 [hep-th/9609070] [INSPIRE]. 
[21] M.R. Douglas, S.H. Katz and C. Vafa, Small instantons, Del Pezzo surfaces and type-I-prime theory, Nucl. Phys. B 497 (1997) 155 [hep-th/9609071] [INSPIRE].

[22] K.A. Intriligator, D.R. Morrison and N. Seiberg, Five-dimensional supersymmetric gauge theories and degenerations of Calabi-Yau spaces, Nucl. Phys. B 497 (1997) 56 [hep-th/9702198] [INSPIRE].

[23] O. Bergman, D. Rodríguez-Gómez and G. Zafrir, 5-Brane Webs, Symmetry Enhancement and Duality in 5d Supersymmetric Gauge Theory, JHEP 03 (2014) 112 [arXiv:1311.4199] [INSPIRE].

[24] G. Zafrir, Duality and enhancement of symmetry in 5d gauge theories, JHEP 12 (2014) 116 [arXiv: 1408.4040] [INSPIRE].

[25] F. Benini, R. Eager, K. Hori and Y. Tachikawa, Elliptic genera of two-dimensional $N=2$ gauge theories with rank-one gauge groups, Lett. Math. Phys. 104 (2014) 465 [arXiv: 1305.0533] [INSPIRE].

[26] F. Benini, R. Eager, K. Hori and Y. Tachikawa, Elliptic Genera of $2 d \mathcal{N}=2$ Gauge Theories, Commun. Math. Phys. 333 (2015) 1241 [arXiv:1308.4896] [InSPIRE].

[27] O. Bergman and G. Zafrir, Lifting 4d dualities to 5d, JHEP 04 (2015) 141 [arXiv: 1410.2806] [INSPIRE]. 